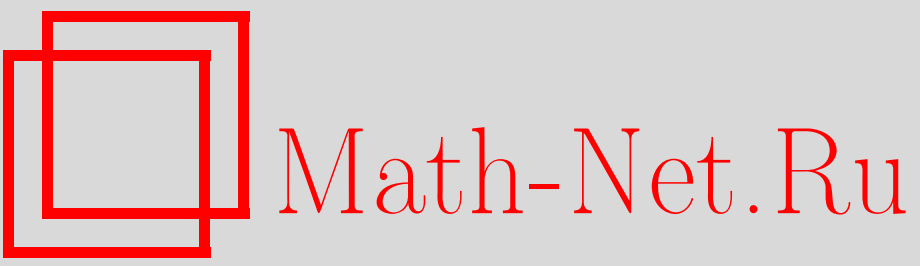

А. А. Гайфуллин, Вычисление характеристических классов многообразия по его триангуляции, УМH, 2005, том 60, выпуск 4, 37-66

DOI: https://doi.org/10.4213/rm1444

Использование Общероссийского математического портала Math-Net.Ru подразумевает, что вы прочитали и согласны с пользовательским соглашением

http://www.mathnet.ru/rus/agreement

Параметры загрузки:

IP: 18.208 .226 .222

26 апреля 2023 г., 18:20:50 


\title{
ВЫЧИСЛЕНИЕ ХАРАКТЕРИСТИЧЕСКИХ КЛАССОВ МНОГООБРАЗИЯ ПО ЕГО ТРИАНГУЛЯЩИИ
}

\begin{abstract}
А. А. ГАЙФУллин
Настоящий обзор посвящен известной проблеме: вычислению характеристических классов Штифеля-Уитни и Понтрягина многообразия по его триангуляции. Локальную комбинаторную формулу для классов Штифеля-Уитни построил Х. Уитни еще в 1940 г. В 1975 г. в работе А. М. Габриэлова, И. М. Гелњфанда и М. В. Лосика впервые была предъявлена комбинаторная формула для первого рационального класса Понтрягина. С тех пор разными авторами было получено несколько различных формул для вычисления рациональных характеристических классов триангулированных многообразий, но ни одна из них не давала алгоритма для вьгисления характеристического цикла только по триангуляции многообразия. В настоящем обзоре описьвается построенная недавно автором новая локалшная комбинаторная формула для первого класса Понтрягина, которая дает такой алгоритм. Этот результат явился следствием решения следующей задачи: построить функцию на множестве классов изоморфизма трехмерных PL-сфер такую, что для любого комбинаторного многообразия цепь, составленная из всех его симплексов коразмерности 4 с коэффициентами, равными значениям данной функции на линках этих симплексов, является циклом.

Библиография: 38 названий.
\end{abstract}

\section{СОДЕРЖАНИЕ}

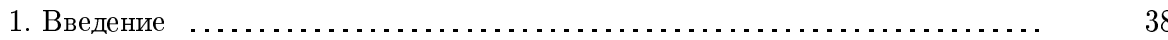

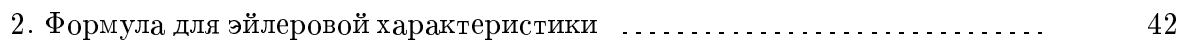

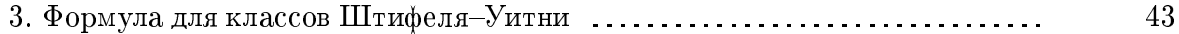

4. Формула Габриэлова-Гельфанда-Лосика ......................... 43

5. Формула Чигера .............................................. 46

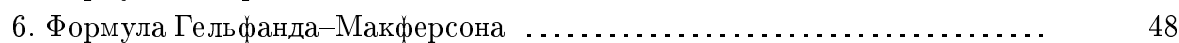

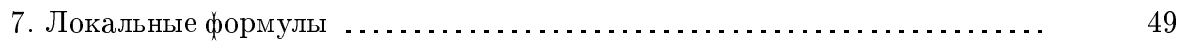

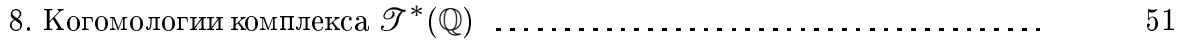

9. Бизвездные преобразования ….............................. 52

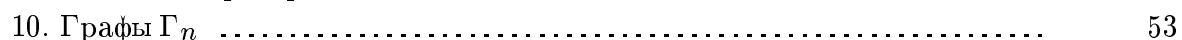

11. Локальные формулы для первого класса Понтрягина .............. 55

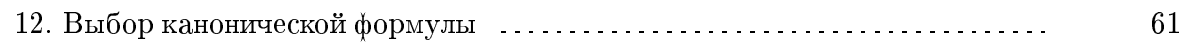

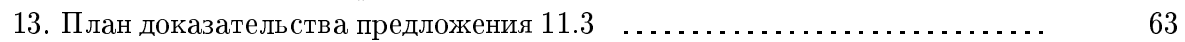

14. Знаменатели значений локальных формул ....................... 64

15. Существование алгоритмов для вьчисления локальных формул ........ 64

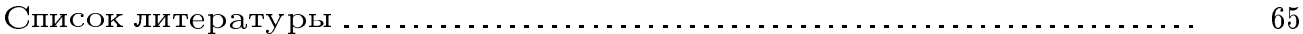


1. Введение. В центре внимания данной работы находятся классы ШтифеляУитни и Понтрягина многообразий. Как известно, определение этих характеристических классов существенно использует гладкую структуру. Тем не менее вскоре после введения классов Штифеля-Уитни была установлена их комбинаторная инвариантность. Комбинаторная инвариантность рациональных классов Понтрягина была доказана в конце 1950-х годов независимо В. А. Рохлиным и А. С. Шварцем [1] и Р. Томом [2]. Однако предложенное ими доказательство неконструктивно, т.е. не позволяет непосредственно вычислять классы Понтрягина по заданной триангулящии многообразия. Поэтому оставалась нерешенной важная задача - получение комбинаторных формул для характеристических классов, т.е. описание способов построения по триангуляции многообразия симплициальных циклов, классы гомологий которых двойственны по Пуанкаре данным характеристическим классам многообразия.

Тесно связанной с предыдушей является задача вычисления чисел Штифеля-Уитни и чисел Понтрягина многообразия по его триангулящии. Здесь сразу необходимо выделить знаменитьй $L$-род Хирцебруха - линейную комбинацию чисел Понтрягина ориентированного $4 k$-мерного многообразия, дающую его сигнатуру. Таким образом, $L$-род допускает комбинаторное описание, так как кольцо когомологий триангулированного многообразия может быть вычислено комбинаторно. Более эффективньй способ вычисления сигнатуры триангулированного многообразия был предложен Э. Раницким и Д. Сулливаном [3]. Они построили симметрическую билинейную форму на прямой сумме груп $2 k$-мерных и $(2 k+1)$-мерных симплициальных цепей $4 k$-мерного триангулированного многообразия такую, что сигнатура этой билинейной формы равна сигнатуре многообразия.

Особый интерес представляет задача построения локальных формул для характеристических классов. Формула назьвается локальной, если в построенном по ней цикле коэффициент при каждом симплексе зависит только от строения многообразия в окрестности этого симплекса. Для рациональных классов Понтрягина часто удается получить более сильное условие локальности: коэффициент при симплексе определяется только комбинаторным строением его линка.

Еще в 1940 году Х. Уитни [4] получил явную комбинаторную формулу для классов Штифеля-Уитни. Она оказалась очень простой. Чтобы получить цикл, двойственньй по Пуанкаре $n$-му классу Штифеля-Уитни $m$-мерного комбинаторного многообразия $K$, нужно просто взять сумму по модулю 2 всех $(m-n)$-мерных симплексов его первого барицентрического подразделения $K^{\prime}$ (см. п. 3).

Все известные комбинаторные формулы для рациональных классов Понтрягина гораздо сложнее. До недавнего времени можно было выделить два основных пути построения комбинаторных формул для классов Понтрягина. Первьй из них был предложен А.М. Габриэловьм, И. М. Гельфандом и М. В. Лосиком [5], [6] и развит Р. Д. Макферсоном [7], А. М. Габриэловым [8] и И. М. Гельфандом и Р. Д. Макферсоном [9]. Различные формулы, полученные в этих работах, используют определения рациональных классов Понтрягина через кривизну связности, через вырождения в системах сечений касательного расслоения и в терминах отображения Гаусса. Подробнее этот подход описан в пп. 4, 6. Второй путь предложил Дж. Чигер [10]

Работа выполнена при финансовой поддержке Российского фонда фундаментальных исследований (грант № 02-01-00659-а) и Государственной программы поддержки ведущих научных школ (грант НШ-2185.2003.1). 
(см. п. 5). Он основан на конструкции $\eta$-инварианта $(4 k-1)$-мерного риманова многообразия, предложенной М.Ф. Атьей, В.К. Патоди и И.М. Зингером [11]. Недавно автором [12] был предложен новый подход к построению комбинаторных формул для классов Понтрягина, основанный на использовании техники бизвездных преобразований. Полученная этим методом локальная комбинаторная формула для первого класса Понтрягина описана в пп. 7-13.

Обсудим теперь более подробно, что подразумевается под словами комбинаторная формула. На самом деле в это словосочетание часто вкладьваются два различных смысла. Во-первых, комбинаторная формула сопоставляет каждому комбинаторному многообразию симплициальный цикл, определяемый только комбинаторным строением данного многообразия. Иногда комбинаторная формула может быть применена только для комбинаторных многообразий, удовлетворяющих некоторым дополнительным условиям. При этом построенньй цикл может быть симплициальньм циклом в исходной триангуляции или в некотором ее подразделении. Во-вторых, часто предполагается, что комбинаторная формула должна задавать алгоритм, которьй вычисляет искомый симплициальный цикл только по заданной триангуляции многообразия. Такие формулы мы будем назьвать алгоритмически вычислимыми.

В настояшее время известны следующие комбинаторные формулы для классов Понтрягина.

1) Модификация Макферсона [7] формулы Габриэлова-Гельфанда-Лосика [6] для первого рационального класса Понтрягина (см. п. 4). Может быть применена для комбинаторных многообразий, удовлетворяюших некоторьм дополнительным условиям, которые выделяют класс так называемых многообразий Брауэра (определение см. в п. 4). Построенньй цикл является симплициальным в исходной триангулящии, и коэффициент при каждом симплексе определяется только комбинаторньм строением линка этого симплекса. Формула не является алгоритмически вычислимой, так как вычисление по ней требует операций с некоторыми сложными пространствами конфигураций. В настояшее время не известно никакого алгоритмического описания этих пространств в комбинаторных терминах.

2) Формула Чигера [10] для всех полиномов Хирцебруха от вешественных классов Понтрягина (см. п. 5). Может быть применена для любого псевдомногообразия с пренебрежимой границей (определение см. в п. 5), в частности, для любого комбинаторного многообразия. Построенньй цикл является симплищиальным в исходной триангулящии, и коэффищиент при каждом симплексе определяется только комбинаторным строением линка этого симплекса. Вычисление по этой формуле сводится к вычислению спектра оператора Лапласа на псевдомногообразиях с локально плоской метрикой. Такое вьчисление может быть произведено только приближенно, поэтому формула Чигера не является алгоритмически вычислимой и неизвестно, является ли построенный по ней цикл рациональным.

3) Формула Гельфанда-Макферсона [9] для всех рациональных нормальных классов Понтрягина (см. п. 6). Эта формула не является комбинаторной в первом смысле, т.е. получаемый по ней цикл зависит не только от комбинаторного строения заданного триангулированного многообразия. Чтобы эта формула могла быть применена для триангулированого многообразия, это многообразие должно быть снабжено дополнительно гладкой структурой или ее дискретньм аналогом - фиксирующим ииклом. Построенньй цикл является симплищиальным в первом барицентрическом подразде- 
лении исходной триангуляции. Коэффищиент при каждом симплексе зависит от комбинаторного строения окрестности этого симплекса и от ограничения гладкой структуры или фиксируюшего цикла на эту окрестность. При заданной гладкой структуре или при заданном фиксирующем цикле вычисление искомого цикла производится с помощью чисто комбинаторной процедуры.

4) Формула автора [12] для первого рационального класса Понтрягина. Формула может быть применена для любого комбинаторного многообразия без каких бы то ни было дополнительных структур. Это единственная известная формула, которая является и локальной, и комбинаторной в первом смысле, и алгоритмически выгислимой. Построенньй цикл является симплициальньм в исходной триангуляции. Коэффициент при каждом симплексе зависит только от комбинаторного строения его линка и может быть вычислен по комбинаторному типу его линка с помощью конечной комбинаторной процедуры.

Основой для сопоставления перечисленных формул является следующее. При построении комбинаторных формул обычно выбирается какое-либо определение характеристических классов гладкого многообразия. При этом сложилось два подхода. Первый, назовем его алгебро-топологическим, использует средства алгебраической топологии, включая гладкость многообразия. Так в оригинальной работе Л. С. Понтрягина [13] характеристический цикл представляет собой цикл особенностей набора из $k$ векторных полей на многообразии. Второй подход - дифференциально-геометрический - использует дифференциально-геометрическую связность на многообразии и ее кривизну (в литературе этот подход часто назьвают подходом Чженя-Вейля). Дифференциально-геометрическое построение характеристических классов вешественных римановых многообразий было проведено Л. С. Понтрягиным [14], [15], характеристических классов комплексных эрмитовых многообразий - Ш.-Ш. Чженем [16].

Как отметил В.М. Бухштабер, результаты автора [12] реализуют новьй, третий подход к построению характеристических классов многообразий. При этом подходе $n$-мерный характеристический класс $m$-мерного триангулированного многообразия $K$ задается универсальной формулой вида

$$
f_{\sharp}(K)=\sum_{\sigma^{m-n} \in K} f\left(\operatorname{link} \sigma^{m-n}\right) \sigma^{m-n},
$$

где $f$ - выбранная функция на классах изоморфизма ориентированных $(n-1)$-мерных PL-cфер. Свойство универсальности заключается в том, что функция $f$ не зависит от комбинаторного многообразия $K$, а цеп $f_{\sharp}(K)$ является циклом для любого комбинаторного многообразия $K$. Более точные определения даны в п. 7. Базисньй результат заключается в том, что для любого рационального характеристического класса сушествует формула указанного вида. Этот результат представляет собой усиление результата Н. Левитта и К. Рурка [17] (см. п. 8). Функция $f$ назьвается локальной формулой, если цеп $f_{\sharp}(K)$ является циклом для любого комбинаторного многообразия $K$. При $n=4$ каждая рациональная локальная формула задает первьй класс Понтрягина с точностью до умножения на некоторую рациональную константу. С другой стороны, оказьвается, что при $n=4$ все локальные формулы могут быть явно описаны с использованием техники бизвездных преобразований. Это сделано в пп. 9-11. Таким образом, получается явное описание всех локальных формул 
для первого рационального класса Понтрягина (теорема 11.1). В п. 12 выделена одна каноническая локальная формула $f_{0}$ для первого класса Понтрягина и описана комбинаторная процедура вычисления коэффициента $f_{0}(L)$ по заданной ориентированной трехмерной PL-сфере $L$.

Хорошо известно, что существуют комбинаторные многообразия, рациональные классы Понтрягина которых не могут быть реализованы целочисленными коциклами. Однако для $k$-го класса Понтрягина существует универсальная константа $N_{k}$ такая, что класс $N_{k} p_{k}(K)$ может быть представлен целочисленньм коциклом для любого комбинаторного многообразия $K$. Рассмотрим другую задачу. Предположим, что мы для каждого комбинаторного многообразия $K$ реализовали класс гомологий, двойственный некоторому заданному рациональному классу Понтрягина многообразия $K$, циклом вида $f_{\sharp}(K)$, где $f$ - функция, не зависящая от многообразия $K$. Какие знаменатели возникают у коэффициентов циклов $f_{\sharp}(K)$, т.е. у значений $f(L)$ ? Оказьвается, что уже для первого класса Понтрягина знаменатели значений $f(L)$ неограниченно возрастают при увеличении числа вершин PL-сфферы $L$. Более того, каждое простое число входит в знаменатели значений $f(L)$ в сколь угодно больших степенях. Более точные оценки на рост знаменателей значений $f(L)$ даны в п. 14 .

В п. 15 приведена теорема, утверждающая, что для любого рационального характеристического класса сушествует локальная формула $f$ такая, что задача вьгисления значения $f(L)$ по заданной PL-сфере $L$ алгоритмически разрешима. Полные доказательства результатов пп. 14, 15 даны автором в [12].

Задача нахождения комбинаторных формул, очевидно, имеет смысл только для комбинаторно инвариантных характеристических классов. В частности, задача о нахождении комбинаторных формул для целочисленных классов Понтрягина является некорректной. Однако каждый целочисленный класс Понтрягина становится комбинаторно инвариантным после умножения на некоторую натуральную константу, поэтому соответствующая задача о нахождении комбинаторной формулы может быть поставлена, хотя никаких таких комбинаторных формул до сих пор не найдено.

В этой статье, если не оговорено противное, все многообразия и триангуляции предполагаются кусочно линейными. Под кобордизмом всегда понимается ориентированньй кусочно линейный кобордизм. Симплициальный комплекс назьвается PL-cферой, если некоторое его подразделение изоморфно некоторому подразделению границы симплекса. Симплищиальный комплекс назьвается $m$-мернымм комбинаторным многообразием, если линк каждой его вершины является $(m-1)$-мерной PL-сферой. Отметим, что любая кусочно линейная триангулящи кусочно линейного многообразия является комбинаторньм многообразием. Все многообразия предполагаются замкнутыми. Под изоморфизмом ориентированных симплициальных комплексов понимается симплициальньй изоморфизм, сохраняющий ориентацию. Будем обозначать через $C K$ конус над симплициальным комплексом $K$, через $K * L$ - соединение (джойн) симплициальных комплексов $K$ и $L$. Будем обозначать через $\operatorname{link} \sigma$ и $\operatorname{star} \sigma$ соответственно линк и звезду симплекса $\sigma$.

Пусть $K-m$-мерное комбинаторное многообразие. Коориентацией симплекса $\sigma^{n} \in K$ назьвается ориентация линка симплекса $\sigma^{n}$. Любой $m$-мерньй симплекс считается положительно коориентированным. Пусть $G$ - абелева групша, $\widehat{G}$ - ориентирующий пучок многообразия $|K|$ со слоем, изоморфньм $G$. Обозначим через $\widehat{C}_{*}(K ; G)$ цепной комплекс коориентированных цепей комплекса $K$ с коэффициентами в $G$, че- 
рез $\widehat{\partial}$ - граничный оператор этого комплекса (коэффициент инщидентности двух коориентированных симплексов $\tau^{k-1} \subset \sigma^{k}$ равен +1 , если ориентация link $\sigma^{k}$ индуцирована ориентацией link $\tau^{k-1}$, и -1 в противном случае). Гомологии комплекса $\widehat{C}_{*}(K ; G)$ совпадают с гомологиями $H_{*}(|K| ; \widehat{G})$. Классы гомологий, двойственные по Пуанкаре рациональным классам Понтрягина многообразия $|K|$, лежат в групе $H_{*}(|K|$; $\widehat{\mathbb{Q}})$. Поэтому представляющие их симплициальные циклы должны лежать в группе $\widehat{C}_{*}(K ; \mathbb{Q})$.

2. Формула для эйлеровой характеристики. В этом пункте рассматривается локальная формула для эйлеровой характеристики конечного симплициального комплекса. Этот пример можно рассматривать как иллюстрацию к определению локальной формулы, которое будет дано в п. 7.

Пусть $L$ - конечньй симплициальньй комплекс. Будем обозначать через $f_{k}(L)$ число $k$-мерных симплексов комплекса $L$. Положим

$$
F(L)=1-\frac{f_{0}(L)}{2}+\frac{f_{1}(L)}{3}-\cdots+\frac{(-1)^{k} f_{k-1}(L)}{k+1}+\cdots
$$

ПРЕДЛОЖЕНИЕ 2.1. Для любого конечного симплициального комплекса $K$ имеет место формула

$$
\chi(K)=\sum_{v \in K} F(\operatorname{link} v)
$$

где сумммирование ведется по всем вершинам $v$ комплекса $K$.

ДокАЗАТЕльство. Каждый $k$-мерный симплекс $\sigma^{k}$ комплекса $K$ имеет ровно $k+1$ вершину. Поэтому число $f_{k}(K)$ равно в $k+1$ раз меньше, чем число пар $\left(v, \sigma^{k}\right)$, где $v-$ вершина комплекса $K, \sigma^{k}$ - симплекс комплекса $K$, причем $v \in \sigma^{k}$. С другой стороны, каждая вершина $v$ содержится ровно в $f_{k-1}(\operatorname{link} v)$ симплексах $\sigma^{k} \in K$. Поэтому

$$
f_{k}(K)=\frac{1}{k+1} \sum_{v \in K} f_{k-1}(\operatorname{link} v)
$$

Значит,

$$
\chi(K)=\sum_{k=0}^{\infty}(-1)^{k} f_{k}(K)=\sum_{k=0}^{\infty} \sum_{v \in K} \frac{(-1)^{k} f_{k-1}(\operatorname{link} v)}{k+1} .
$$

Если $K$ - комбинаторное многообразие, то характеристический цикл, задающий его гомологический эйлеров класс, может быть вычислен с помощью локальной формулы

$$
E(K)=\sum_{v \in K} F(\operatorname{link} v) v
$$

Обратим внимание, что в представлении целочисленного класса Эйлера с помошњю локальной формулы у коэффициентов $F(\operatorname{link} v)$ возникают сколь угодно большие знаменатели, которые исчезают при переходе к нелокальной формуле. 
3. Формула для классов Штифеля-Уитни. Пусть $K$ - комбинаторное многообразие размерности $m$. Его класс Штифеля-Уитни $w_{n}$ лежит в когомологиях с коэффициентами в $\mathbb{Z}_{2}$, если $n$ четно, и с коэффициентами в $\widehat{\mathbb{Z}}$, если $n$ нечетно, где $\widehat{\mathbb{Z}}$ - ориентируюший пучок многообразия $|K|$ со слоем $\mathbb{Z}$. Обозначим через $W_{n}$ класс гомологий, двойственньй классу $w_{n}$. Тогда $W_{n} \in H_{m-n}\left(|K| ; \mathbb{Z}_{2}\right)$, если $n$ четно, и $W_{n} \in H_{m-n}(|K| ; \mathbb{Z})$, если $n$ нечетно. Обозначим через $K^{\prime}$ первое барищентрическое подразделение симплициального комплекса $K$. Если $n$ четно, обозначим через $C_{n}$ сумму по модулю 2 всех $(m-n)$-мерных симплексов комплекса $K^{\prime}$. Пусть теперь $n$ нечетно. Пусть $\sigma_{0} \subset \sigma_{1} \subset \cdots \subset \sigma_{m-n}-$ различные непустые симплексы комплекса $K$. Обозначим через $\tau\left(\sigma_{0}, \sigma_{1}, \ldots, \sigma_{m-n}\right)$ симплекс комплекса $K^{\prime}$ с вершинами в барицентрах $b\left(\sigma_{i}\right)$ симплексов $\sigma_{i}$. Ориентация симплекса $\tau\left(\sigma_{0}, \sigma_{1}, \ldots, \sigma_{m-n}\right)$ задается последовательностью вершин $b\left(\sigma_{0}\right), b\left(\sigma_{1}\right), \ldots, b\left(\sigma_{m-n}\right)$. Определим целочисленную $(m-n)$-мерную цепь $C_{n}$ по формуле

$$
C_{n}=\sum_{\sigma_{0} \subset \sigma_{1} \subset \cdots \subset \sigma_{m-n}}(-1)^{\operatorname{dim} \sigma_{0}+\operatorname{dim} \sigma_{1}+\cdots+\operatorname{dim} \sigma_{m-n}} \tau\left(\sigma_{0}, \sigma_{1}, \ldots, \sigma_{m-n}\right) .
$$

ТеОРема 3.1. Цепь $C_{n}$ является ииклом (по модулю 2 , если п четно, и иелочисленным, если п нечетно), представляющим класс гомологий $W_{n}$, двойственный по Пуанкаре $n$-му классу Штифеля-Уитни многообразия $K$.

Эта теорема была сформулирована в качестве гипотезы Э. Штифелем [18] и впервые доказана Х. Уитни [4], однако полное доказательство не было опубликовано. Для гладких многообразий полное доказательство было впервые опубликовано С. Гальпериным и Д. Толедо [19]. Их доказательство основано на явном построении непрерьвных касательных векторных полей $F_{1}, \ldots, F_{m}$ на $|K|$, гладких на каждом симплексе, таких, что $F_{1}, \ldots, F_{p}$ линейно независимы на каждом $p$-мерном симплексе $\sigma \in K$ и индекс поля $F_{p+1}\left(\bmod F_{1}, \ldots, F_{p}\right)$ в барищентре симплекса $\sigma$ равен \pm 1 . На самом деле это доказательство работает для произвольного комбинаторного многообразия. Набросок альтернативного доказательства теоремы 3.1, используюшего совсем другую технику, был опубликован Дж. Чигером [20].

4. Формула Габриэлова-Гельфанда-Лосика. Первая явная формула для первого ращионального класса Понтрягина триангулированного многообразия была получена А.М. Габриэловым, И. М. Гельфандом и М. В. Лосиком [5]. Опишем здесь некоторые идеи, лежашие в основе этой формулы. Пусть $M$ - гладкое многообразие размерности $m, K$ - его гладкая триангуляция. Пусть $\nabla$ - гладкая связность в касательном расслоении многообразия $M$. Если задана локальная тривиализация касательного расслоения, то связность $\nabla$ задается 1-формой $\omega$ со значениями в $\mathfrak{g l}(m, \mathbb{R})$. Пусть $\Omega=d \omega-\omega \wedge \omega-$ форма кривизны. Тогда

$$
P^{0}(\nabla)=\operatorname{tr}(\Omega \wedge \Omega)=\operatorname{tr}(d \omega \wedge d \omega-2 \omega \wedge \omega \wedge d \omega)
$$

- корректно определенная 4-форма на многообразии $M$. Хорошо известно, что значение первого класса Понтрягина многообразия $M$ на кусочно гладком 4-мерном цикле $Z$ выражается следующей формулой.

$$
\left\langle p_{1}(M), Z\right\rangle=-\frac{1}{8 \pi^{2}} \int_{Z} P^{0}(\nabla)
$$


Пусть $\tau \in K$ - коориентированный симплекс размерности $m-1$. Обозначим через $\sigma_{0}(\tau)$ и $\sigma_{1}(\tau)$ два содержаших его $m$-мерных симплекса. Пусть $\rho \in K$ - коориентированньй симплекс размерности $m-2$. Обозначим через $\sigma_{1}(\rho), \ldots, \sigma_{k(\rho)}(\rho)$ содержашие его $m$-мерные симплексы, занумерованные в направлении положительного обхода линка симплекса $\rho$.

ПРЕДЛОЖЕНИЕ 4.1. Пусть на каждом гладкая связность $\nabla_{\sigma}$ в касательном расслоении многообразия $K$. Пусть $Z$ кусочно гладкий 4-мерный иикл, трансверсальный к триангуляиии K. Тогда

$$
\begin{aligned}
\left\langle p_{1}(M), Z\right\rangle=- & \frac{1}{8 \pi^{2}}\left(\sum_{\operatorname{dim} \sigma=m} \int_{Z \cap|\sigma|} P^{0}\left(\nabla_{\sigma}\right)\right. \\
& +\sum_{\operatorname{dim} \tau=m-1} \int_{Z \cap|\tau|} P^{1}\left(\nabla_{\sigma_{0}(\tau)}, \nabla_{\sigma_{1}(\tau)}\right) \\
& \left.+\sum_{\operatorname{dim} \rho=m-2} \sum_{j=2}^{k(\rho)-1} \int_{Z \cap|\rho|} P^{2}\left(\nabla_{\sigma_{1}(\rho)}, \nabla_{\sigma_{j}(\rho)}, \nabla_{\sigma_{j+1}(\rho)}\right)\right),
\end{aligned}
$$

әде

$$
\begin{gathered}
P^{1}\left(\nabla_{0}, \nabla_{1}\right)=\operatorname{tr}\left(\left(\omega_{1}-\omega_{0}\right) \wedge d\left(\omega_{1}+\omega_{0}\right)+\frac{2}{3} \omega_{0} \wedge \omega_{0} \wedge \omega_{0}-\frac{2}{3} \omega_{1} \wedge \omega_{1} \wedge \omega_{1}\right) \\
P^{2}\left(\nabla_{0}, \nabla_{1}, \nabla_{2}\right)=-\operatorname{tr}\left(\omega_{0} \wedge \omega_{1}+\omega_{1} \wedge \omega_{2}+\omega_{2} \wedge \omega_{0}\right) .
\end{gathered}
$$

Здесь $\omega_{i}-1$-формы со значениями в $\mathfrak{g l}(m, \mathbb{R})$, соответствуюшие связностям $\nabla_{i}$ при некотором выборе локальной тривиализачии.

Набор форм $P^{0}(\nabla), P^{1}\left(\nabla_{0}, \nabla_{1}\right), P^{2}\left(\nabla_{0}, \nabla_{1}, \nabla_{2}\right)$ называется разностным кочиклом, так как

$$
\begin{gathered}
d P^{1}\left(\nabla_{0}, \nabla_{1}\right)=P^{0}\left(\nabla_{1}\right)-P^{0}\left(\nabla_{0}\right) \\
-d P^{2}\left(\nabla_{0}, \nabla_{1}, \nabla_{2}\right)=P^{1}\left(\nabla_{0}, \nabla_{1}\right)+P^{1}\left(\nabla_{1}, \nabla_{2}\right)+P^{1}\left(\nabla_{2}, \nabla_{0}\right) .
\end{gathered}
$$

Выберем в качестве $\nabla_{\sigma}$ связности нулевой кривизны такие, что, во-первых, для любого симплекса $\tau \subset \sigma$ касательное расслоение $T|\tau|$ симплекса $\tau$ инвариантно относительно $\nabla_{\sigma}$, а во-вторых, для любых $m$-мерных симплексов $\sigma_{1}$ и $\sigma_{2}$ ограничения связностей $\nabla_{\sigma_{1}}$ и $\nabla_{\sigma_{2}}$ на $T\left|\sigma_{1} \cap \sigma_{2}\right|$ совпадают. Тогда первые два слагаемые в правой части формулы (*) будут равны нулю. Таким образом, вычисление интеграла по 4-мерному циклу $Z$ сводится к вычислению интегралов от некоторых 2-форм по 2-мерньм цепям $Z \cap|\rho|$. Следуюшие шаги - свести вычисление этих интегралов сначала к вычислению интегралов от некоторых 1-форм по пересечениям цикла $Z$ с симплексами коразмерности 3 , а потом - к нахождению индекса пересечения цикла $Z$ с некоторым $(m-4)$-мерным циклом, который и будет искомым циклом, класс гомологий которого двойствен классу $p_{1}(M)$. Однако эти шаги вьполняются намного сложнее и требуют изучения топологии пространств конфигураций $\Sigma(\sigma)$. Мы здесь не будем приводить полного построения формулы работы [5], однако дадим определения пространств $\Sigma(\sigma)$ и укажем, какие именно их свойства используются при построении формулы. 
ОПРЕДЕЛЕНИЕ 4.1. Сглаживанием комбинаторного многообразия $K$ в симплексе $\sigma$ назьвается гомеоморфизм из $|C \operatorname{link} \sigma|$ на окрестность начала координат в $\mathbb{R}^{\operatorname{codim} \sigma}$, который переводит вершину конуса в начало координат и линеен на каждом симплексе. Комбинаторное многообразие $K$ назьвается многообразием Брауәра, если для любого непустого симплекса $\sigma \in K$ существует сглаживание многообразия $K$ в симплексе $\sigma$.

Не любое комбинаторное многообразие является многообразием Брауэра [21], но для любого компактного комбинаторного многообразия некоторое его барицентрическое подразделение является многообразием Брауэра [22]. Не любое многообразие Брауэра является сглаживаемым: чтобы комбинаторное многообразие было сглаживаемьм, необходимо, чтобы его сглаживания в симплексах можно было выбрать согласованным образом.

Пусть $K$-многообразие Брауэра, $\sigma \in K$ - симплекс коразмерности $k$. Пространство сглаживаний многообразия $K$ в симплексе $\sigma$ является топологическим пространством с действием групшы $G L(k, \mathbb{R})$. Пространство орбит этого действия обозначается через $\Sigma(\sigma)$ и называется пространством конфигураиий. Несложно доказать, что $\Sigma(\sigma)$ стягиваемо, если $\operatorname{codim} \sigma=2$. Известно также, что $\Sigma(\sigma)$ связно [23] и односвязно [24], если $\operatorname{codim} \sigma=3$. Формула работы [5] может быть применена только для таких триангуляций $K$, что выполнено так называемое условие (А): пространство $\Sigma(\sigma)$ связно для любого $\sigma \in K$ такого, что $\operatorname{codim} \sigma=4$. Неизвестно, для каких классов триангулящий это условие вьполнено.

Пусть $q$ - количество вершин комплекса $\operatorname{link} \sigma$. Тогда каждому сглаживанию $\psi:|C \operatorname{link} \sigma| \rightarrow \mathbb{R}^{k}$ соответствует набор из $q$ ненулевых векторов в $\mathbb{R}^{k}$. Пространство $\Sigma(\sigma)$ имеет естественную стратификацию: оно представляется в виде объединения непересекаюшихся стратов $\Sigma_{c}(\sigma), c=0,1,2, \ldots$, где $\Sigma_{0}(\sigma)$ состоит из классов эквивалентности сглаживаний, для которых соответствуюшая конфигурация из $q$ векторов в $\mathbb{R}^{k}$ находится в общем положении, т.е. не содержит $k$ линейно зависимых векторов, а $\Sigma_{c}(\sigma), c>0$, состоит из классов эквивалентности сглаживаний, для которых соответствуюшая конфигурация векторов имеет $c$-кратное вырождение.

Формула, полученная в [5], может быть применена к любому многообразию Брауэра, удовлетворяющему условию (А). Для вычисления по этой формуле необходимо на многообразии $K$ выбрать следуюшие дополнительные структуры: для каждого симплекса $\tau$ коразмерности 4 - точку $y_{\tau} \in \Sigma_{0}(\tau)$, для каждого симплекса $\sigma$ коразмерности 3 - точку $y_{\sigma} \in \Sigma_{0}(\sigma)$, для каждой пары симплексов $\tau \subset \sigma, \operatorname{codim} \tau=4$, $\operatorname{codim} \sigma=3,-$ кривую $z_{\sigma, \tau}(t)$ в $\Sigma_{0}(\sigma) \cup \Sigma_{1}(\sigma)$ такую, что $z_{\sigma, \tau}(0)=y_{\sigma}, z_{\sigma, \tau}(1)-$ образ точки $y_{\tau}$ при естественном отображении $\Sigma(\tau) \rightarrow \Sigma(\sigma)$. Кроме того, нужно выбрать некоторую дополнительную комбинаторную структуру, назьваемую гиперсимплициальной системой. После того как все эти структуры выбраны, непосредственным комбинаторньм вычислением получается рациональньй симплициальный цикл, класс гомологий которого двойствен по Пуанкаре первому классу Понтрягина многообразия $K$. Заметим, что стратификации пространств $\Sigma(\rho)$ устроены достаточно сложно и не существует никакого комбинаторного способа выбора точек $y_{\sigma}, y_{\tau}$ и кривых $z_{\sigma, \tau}$. Поэтому формула Габриэлова-Гельфанда-Лосика не является алгоритмически вычислимой. Единственньй случай, когда дополнительные структуры могут быть выбраны некоторьм комбинаторньм способом, - случай, когда задано глобаль- 
ное сглаживание многообразия $K$.

В [8] А.М. Габриэловым получено обобшение формулы работы [5] для старших классов Понтрягина. При этом для вычисления $k$-го класса Понтрягина требуется, чтобы многообразие $K$ удовлетворяло условию $\left(\mathrm{A}_{4 k}\right)$, обобшаюшему условие $(\mathrm{A})$ :

$$
\widetilde{H}_{q}\left(\Sigma_{\sigma} ; \mathbb{Q}\right)=0 \text { при } 0 \leqslant q \leqslant 4 k-\operatorname{codim} \sigma
$$

для любого симплекса $\sigma \in K$. Это условие является очень ограничительным, и не известно никакого способа проверять, вьполняется ли оно для данного многообразия Брауэра. Тем более не существует никакого комбинаторного способа производить вычисления по полученной формуле. Отличие подхода А. М. Габриэлова состоит в том, что вместо определения классов Понтрягина через кривизну связности он использует определение классов Понтрягина через вырождения в системах сечений касательного расслоения (см. [13], [25]).

Формула, полученная в работе [5], не является локальной. В [6] описана процедура усреднения по выбору дополнительных структур, которая позволяет для любого многообразия Брауэра, удовлетворяюшего условию (А), строить рациональньй симплициальньй цикл, класс гомологий которого двойствен классу $p_{1}(|K|)$, так, что коэффициент при каждом симплексе в построенном цикле определяется только комбинаторным строением его линка. Однако для выгислений по этой формуле нам надо еще больше знать о пространствах $\Sigma(\rho)$ : теперь нам нужно не только уметь выбирать какие-нибудь точки $y_{\sigma}, y_{\tau}$ и кривые $z_{\sigma, \tau}$, но и описывать все такие наборы точек и кривых, удовлетворяющие некоторьм специальным условиям.

В работе [7] Р. Д. Макферсон построил модификацию формулы работы [6], позволяюшую отказаться от условия (A). Таким образом, полученная формула может быть применена к произвольному многообразию Брауэра и коэффициент при каждом симплексе в полученном цикле будет зависеть только от комбинаторного строения линка этого симплекса. Однако эта формула по-прежнему содержит шаг, которьй связан с описанием стратификации пространств $\Sigma(\rho)$ и не может быть выполнен комбинаторно. В [7] предложено новое доказательство того, что построенная формула определяет шикл, класс гомологий которого двойствен первому классу Понтрягина. Идея доказательства заключается в том, чтобы построить гомологический аналог отображения Гаусса. Если $M^{m}$ - гладкое многообразие, то для любого вложения $M^{m} \hookrightarrow \mathbb{R}^{n-1}$ определено отображение Гаусса $g: M^{m} \rightarrow G_{n-1, m}$, где $G_{n-1, m}$ - многообразие Грассмана $m$-мерных подпространств в $\mathbb{R}^{n-1}$. Взяв композицию отображения $g$ с естественным вложением $G_{n-1, m} \hookrightarrow G_{n, m+1}$, получим отображение $g_{1}: M^{m} \rightarrow G_{n, m+1}$. При этом $g^{*} \gamma_{n-1}^{m} \cong T M^{m}$ и $g_{1}^{*} \gamma_{n}^{m+1} \cong T M^{m} \oplus \varepsilon^{1}$, где $T M^{m}$ - касательное расслоение многообразия $M^{m}, \gamma_{n}^{m}$ - тавтологическое расслоение над $G_{n, m}, \varepsilon^{1}$ - тривиальное одномерное расслоение. Пусть теперь $K$ - многообразие Брауэра размерности $m$ с $n$ вершинами. Тогда определено стандартное вложение $|K| \hookrightarrow \Delta^{n-1} \subset \mathbb{R}^{n-1}$. Р. Макферсон явно строит гомологическое 4-отображение Гаусса, т.е. цепное отображение $f: C_{i}\left(K^{*} ; \mathbb{Q}\right) \rightarrow C_{i}\left(G_{n, m+1} ; \mathbb{Q}\right), i \leqslant 4$, такое, что $f^{*} p_{1}\left(\gamma_{n}^{m+1}\right)=p_{1}(|K|)$. Здесь $K^{*}-$ клеточное разбиение многообразия $|K|$, двойственное триангуляции $K$.

5. Формула Чигера. Подход Дж. Чигера [10] основан на построении теории Ходжа для псевдомногообразий с локально-плоской метрикой, удовлетворяющих некоторьм локальным топологическим условиям. Симплициальньй комплекс размерности 
$m$ называется псевдомногообразием, если каждый его симплекс содержится в некотором $m$-мерном симплексе и при этом каждый $(m-1)$-мерньй симплекс содержится ровно в двух $m$-мерных симплексах. Пусть $K$ - компактное псевдомногообразие размерности $m, \Sigma^{m-2}$ - его $(m-2)$-мерный остов. Введем на $|K|$ метрику такую, что ее ограничение на каждьй симплекс совпадает с евклидовой метрикой на правильном симплексе с ребром 1 . Тогда $|K| \backslash\left|\Sigma^{m-2}\right|$ - некомпактное гладкое риманово многообразие. Обозначим через $H_{(2)}^{*}\left(|K| \backslash\left|\Sigma^{m-2}\right| ; \mathbb{R}\right)$ пространства $L_{2}$-когомологий этого многообразия. По определению положим $H_{(2)}^{*}(K ; \mathbb{R})=H_{(2)}^{*}\left(|K| \backslash\left|\Sigma^{m-2}\right| ; \mathbb{R}\right)$. Несложно проверить, что так определенные $L_{2}$-когомологии псевдомногообразия $K$ не изменяются при переходе к подразделениям и, следовательно, являются кусочно линейно инвариантными.

ОПРЕДЕЛЕНИЕ 5.1. Псевдомногообразие $K$ называется псевдомногообразием $c$ пренебрежимой границей, если $H_{(2)}^{k}(\operatorname{link} \sigma ; \mathbb{R})=0$ для любого непустого симплекса $\sigma \in K$ такого, что $\operatorname{dim} \operatorname{link} \sigma=2 k$.

Это условие нужно для того, чтобы замыкания операторов $d$ и $d^{*}$ в пространствах $L_{2}$-форм на многообразии $|K| \backslash\left|\Sigma^{m-2}\right|$ были сопряжены. В частности, любое комбинаторное многообразие является псевдомногообразием с пренебрежимой границей. Пространства $L_{2}$-когомологий компактных псевдомногообразий с пренебрежимой границей всегда конечномерны.

Для псевдомногообразия $L$ размерности $4 k-1$ с пренебрежимой границей Дж. Чигер определяет аналог функционала Атьи-Патоди-Зингера $\eta(L)$. Пусть $\varphi_{j}-$ козамкнутые собственные $(2 k-1)$-формы оператора Лапласа на дополнении к $(4 k-3)$-мерному остову псевдомногообразия $L, \mu_{j}$ - соответствующие собственные значения. $Ф$ ормы $\varphi_{j}$ могут быть нормированы условием $d \varphi_{j}= \pm \frac{\sqrt{\mu_{j}}}{2 k-1} * \varphi_{j}$. Рассмотрим функцию

$$
\eta(s)=\int_{|L|} \frac{2}{\sqrt{\pi}} \sum_{j} \mu_{j}^{-\left(s+\frac{1}{2}\right)} \varphi_{j} \wedge d \varphi_{j}, \quad \operatorname{Re} s<-\frac{1}{2} .
$$

Эта функция продолжается до мероморфной на всей комплексной плоскости функции, не имеющей полюса в точке 0 . Тогда $\eta(L)=\eta(0)$. Заметим, что значение $\eta(L)$ вещественно и зависит только от комбинаторного типа псевдомногообразия $L$.

Теорема 5.1 (Дж. Чигер [10]). Пусть $K$ - ориентируемое псевдомногообразие размерности т с пренебрежимой гранищей. Тогда цепь

$$
c_{m-4 k}(K)=\sum_{\sigma^{m-4 k} \in K} \eta\left(\operatorname{link} \sigma^{m-4 k}\right) \sigma^{m-4 k}
$$

является ииклом. Класс гомологий иикла $c_{m-4 k}(K)$ не изменяется при переходе к подразделениям псевдомногообразия $K$, следовательно, является кусочно линейным инвариантом. Если $K$ - комбинаторное многообразие, то класс гомологий иикла $c_{m-4 k}(K)$ двойствен по Пуанкаре $4 k$-мерному $L$-классу Хирцебруха многообразия $|K|$.

Классы Понтрягина могут быть представлены как полиномы от $L$-классов Хирцебруха, т.е. $L$-классы Хирцебруха являются альтернативной системой порождающих 
в кольце Понтрягина $\mathbb{Q}\left[p_{1}, p_{2}, \ldots\right]$. Теорема 5.1 может рассматриваться как определение гомологических $L$-классов для псевдомногообразий с пренебрежимой границей. Формула Чигера является комбинаторной в том смысле, что цикл $c_{m-4 k}(K)$ определяется только комбинаторным строением псевдомногообразия $K$. Однако не известно никакого комбинаторного способа вычисления значения $\eta(L)$ по заданному псевдомногообразию $L$. Кроме того, неизвестно, являются ли значения $\eta(L)$ рациональньми. Таким образом, теорема 5.1 задает только вещественные циклы.

6. Формула Гельфанда-Макферсона. В [9] И. М. Гельфанд и Р. Д. Макферсон получили комбинаторные формулы для всех рациональных нормальных классов Понтрягина. Нормальные классы Понтрягина многообразия $M$ - это классы $\tilde{p}_{k}(M) \in$ $H^{4 k}(M ; \mathbb{Q})$ такие, что $\left(1+p_{1}(M)+p_{2}(M)+\cdots\right) \smile\left(1+\tilde{p}_{1}(M)+\tilde{p}_{2}(M)+\cdots\right)=1$. Подход [9] является дискретизацией следующего подхода к определению классов Понтрягина. Пусть $M$ - нечетномерное гладкое многообразие размерности $m, E$ - тотальное пространство расслоения $\eta=T M \oplus \varepsilon^{1}$, где $\varepsilon^{1}$ - тривиальное одномерное расслоение над $M$. Обозначим через $\pi: \mathscr{Y} \rightarrow M$ грассманизацию расслоения $\eta$ со слоем $G_{m+1, m-1}$, через $\xi$ - тавтологическое 2 -мерное расслоение над $\mathscr{Y}$.

ПРЕДЛОЖЕНИЕ 6.1. Нормальные классы Понтрягина многообразия М удовлетворяют равенству

$$
\tilde{p}_{k}(M) \frown[M]=(-1)^{k} \pi_{*}\left(e(\xi)^{m-1+2 k} \frown[\mathscr{Y}]\right),
$$

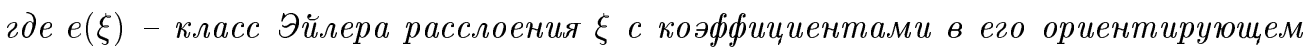
пучке.

Обший подход к построению характеристических классов, основанный на прямом образе эйлерова класса грассманизации исходного расслоения, был дан В.М. Бухштабером в [26] (детальное изложение см. в [27]).

Дискретный аналог описанной конструкции опирается на использование ориентированных матроидов (введение в теорию ориентированных матроидов см. в [28], [29]), Пусть $K$ - нечетномерное ориентированное симплициальное многообразие, $m$ - его размерность. Использование ориентированных матроидов позволяет построить симплициальньй комплекс $Y$ - комбинаторньй аналог пространства $\mathscr{Y}$. Аналогом отображения $\pi$ будет симплициальное отображение $\widehat{\pi}: Y \rightarrow K^{\prime}$, где $K^{\prime}$ - барищентрическое подразделение комплекса $K$. Над $Y$ будет существовать каноническое топологическое $S^{1}$-расслоение, причем рациональный симплициальный коцикл $\Omega$, представляющий класс Эйлера этого $S^{1}$-расслоения, может быть вычислен комбинаторно. При этом комплекс $Y$ и коцикл $\Omega$ вычисляются по триангуляции $K$ локально: строение прообраза $\widehat{\pi}^{-1}(|L|)$ и ограничение $\left.\Omega\right|_{\widehat{\pi}^{-1}(|L|)}$ зависят только от комбинаторного строения многообразия $K$ внутри окрестности подкомплекса $L \subset K$.

Теорема 6.1 (И.М. Гельфанд, Р. Д. Макферсон [9]). Пусть $\varphi \in C_{3 m-2}(Y ; \mathbb{Z})-$ симплициальный ичикл такой, что $\widehat{\pi}_{*}\left(\Omega^{m-1} \frown \varphi\right)=\left[K^{\prime}\right]$. Определим симплициальныци иикл $\zeta_{k} \in C_{m-4 k}\left(K^{\prime} ; \mathbb{Q}\right)$ по формуле

$$
\zeta_{k}=(-1)^{k} \widehat{\pi}_{*}\left(\left(\frac{1}{2} \Omega\right)^{m-1+2 k} \frown \varphi\right) .
$$


Тогда иикл $\zeta_{k}$ представляет класс гомологий, двойственный по Пуанкаре класcy $\tilde{p}_{k}(|K|)$.

Полученная формула легко обощается на случай четномерного многообразия, а также на случай неориентированного многообразия.

Цикл $\varphi$, удовлетворяющий условиям теоремы 6.1, называется фиксирующим ииклом и является аналогом фундаментального цикла многообразия Я . Фиксируюший цикл не может быть выбран канонически, потому что в отличие от У пространство $Y$ уже не будет многообразием. И. М. Гельфанд и Р. Д. Макферсон рассматривают цикл $\varphi$ как структуру на многообразии $K$, являюшуюся комбинаторным аналогом гладкой структуры. Цикл $\varphi$ может быть (локально) восстановлен по заданному глобальному сглаживанию многообразия $|K|$, однако не известно никакого способа построения фиксируюшего цикла для произвольного комбинаторного многообразия $K$. Таким образом, формула Гельфанда-Макферсона может быть применена только для симплициального многообразия с заданным фиксируюшим циклом или сглаживанием, что является ее недостатком по сравнению с формулами работ [7] и [10]. С другой стороны, ее преимушество заключается в том, что если задан фиксирующий цикл или сглаживание, то цикл $\zeta_{k}$ вычисляется с помощью конечной комбинаторной процедуры. При этом цикл $\zeta_{k}$ определяется по триангуляции $K$ и фиксирующему циклу (сглаживанию) локально: коэффициент при каждом симплексе комплекса $K^{\prime}$ в цикле $\zeta_{k}$ зависит только от комбинаторного строения многообразия $K^{\prime}$ в окрестности этого симплекса и от ограничения фиксируюшего цикла (сглаживания) на прообраз этой окрестности.

7. Локальные формулы. В оставшейся части работы будет описана локальная комбинаторная формула для первого класса Понтрягина, полученная автором в [12]. Это первая формула, позволяющая для произвольного комбинаторного многообразия без каких бы то ни было дополнительных структур чисто комбинаторным путем вычислять цикл, класс гомологий которого двойствен первому классу Понтрягина этого многообразия. Полученный цикл будет симплициальньм циклом в исходной триангулящии многообразия, а не в каком-либо ее подразделении. При этом коэффициент при каждом симплексе будет определяться только комбинаторным строением линка этого симплекса. Таким образом, полученный цикл будет иметь вид

$$
f_{\sharp}(K)=\sum_{\sigma^{m-4} \in K} f\left(\operatorname{link} \sigma^{m-4}\right) \sigma^{m-4},
$$

где $m=\operatorname{dim} K, f$ - функщия на классах изоморфизма ориентированных 3 -мерных PL-cфер. При $m>4$ симплексы $\sigma^{m-4} \in K$ не имеют выделенной ориентации, поэтому написанная сумма имеет смысл, только если значение $f(L)$ меняет знак при замене ориентации PL-сферы $L$. В п. 2 была построена функция $F$, задающая локальную формулу для класса Эйлера ориентированного многообразия. При этом значение $F(L)$ не зависит от ориентации $\mathrm{PL}$-сферы $L$. Это различие соответствует тому, что класс Эйлера ориентированного многообразия изменяет знак при замене ориентации многообразия, в то время как классы Понтрягина не зависят от ориентации многообразия. Получение формулы для первого класса Понтрягина распадается на две части: во-первых, используя технику бизвездных преобразований, удается описать все функции $f$ такие, что для любого комбинаторного многообразия цеп $f_{\sharp}(K)$ является шиклом (см. пп. 9-11, 13), во-вторых, оказьвается, что любая такая функция $f$ задает 
цикл, класс гомологий которого двойствен первому классу Понтрягина, умноженному на некоторую константу (п. 8, теорема 8.3).

Дадим теперь точные определения. Пусть $\mathscr{T}_{n}-$ множество всех классов изоморфизма ориентированных $(n-1)$-мерных PL-сфер (считаем, что $\mathscr{T}_{0}=\{\varnothing\}, \mathscr{T}_{-n}=\varnothing$, $n>0)$. Как правило, мы не будем различать PL-сферу и ее класс изоморфизма. Для любой PL-сферы $L \in \mathscr{T}_{n}$ обозначим через $-L$ PL-cферу, полученную из $L$ заменой ориентации. Назовем PL-сферу $L \in \mathscr{T}_{n}$ симметричной, если она изоморфна PL-cфере $-L$. Пусть $G$ - абелева группа. Обозначим через $\mathscr{T}^{n}(G)$ абелеву группу всех функций $f: \mathscr{T}_{n} \rightarrow G$, изменяюших знак при замене ориентации PL-сферы $\left(\mathscr{T}^{0}(G)=G, \mathscr{T}^{-n}(G)=0, n>0\right)$.

Пусть $K-m$-мерное комбинаторное многообразие. Каждой функции $f \in \mathscr{T}^{n}(G)$ сопоставим коориентированную цепь $f_{\sharp}(K) \in \widehat{C}_{m-n}(K ; G)$ по формуле

$$
f_{\sharp}(K)=\sum_{\sigma^{m-n} \in K} f\left(\operatorname{link} \sigma^{m-n}\right) \sigma^{m-n}
$$

(слагаемое $f\left(\operatorname{link} \sigma^{m-n}\right) \sigma^{m-n}$ не зависит от выбора коориентации симплекса $\sigma^{m-n}$ ).

ОПРЕДЕЛЕНИЕ 7.1. Назовем функиию $f \in \mathscr{T}^{n}(G)$ локальной формулой, если для любого комбинаторного многообразия $K$ коориентированная цепь $f_{\sharp}(K)$ является чиклом.

Определим дифференциал $\delta: \mathscr{T}^{n}(G) \rightarrow \mathscr{T}^{n+1}(G)$ по формуле

$$
(\delta f)(L)=\sum f(\operatorname{link} v)
$$

где суммирование ведется по всем вершинам $v$ PL-сферы $L \in \mathscr{T}_{n+1}$ и ориентация $\operatorname{link} v$ индуцирована ориентацией $L$ (как ориентация границы звезды вершины $v$ ). Легко проверить, что $\delta^{2}=0$. Таким образом, в $\mathscr{T}^{*}(G)$ вводится структура коцепного комплекса.

Следующие два предложения доказываются непосредственно.

ПРЕДЛОЖЕНИЕ 7.1. Функиия $f$ является локальной формулой тогда и только тогда, когда $f$ является коциклом в коцепном комплексе $\mathscr{T}^{*}(G)$. Если $f$ является кограницей в коцепном комплексе $\mathscr{T}^{*}(G)$, то для любого комбинаторного многообразия $K$ коориентированная цепь $f_{\sharp}(K)$ является границей.

ПрЕДЛОЖЕНИЕ 7.2. Пусть $f \in \mathscr{T}^{n}(G)$ - локальная формула, $K$ - комбинаторное многообразие с краем. Тогда $\widehat{\partial} f_{\sharp}(K)=i\left(f_{\sharp}(\partial K)\right)$, где $i: \widehat{C}_{*}(\partial K ; G) \rightarrow \widehat{C}_{*}(K ; G)$ - естественное вложсение, $f_{\sharp}(K)$ - чепь, в которую каждый $(\operatorname{dim} K-n)$-мерный симплекс $\sigma \in K$ входит с коэффичиентом $f(\operatorname{link} \sigma)$, если $\sigma$ не содержится в $\partial K$, и с коэффициентом $f\left(\operatorname{link} \sigma \cup_{\partial} \operatorname{link} \sigma C \partial \operatorname{link} \sigma\right)$, если $\sigma$ содержится в $\partial K$.

СлЕДСТВИЕ 7.1. Если $K_{1}$ и $K_{2}$ - две триангулячии многообразия $M^{m}, f \in$ $\mathscr{T}^{n}(G)$ - локальная формула, то чикль $f_{\sharp}\left(K_{1}\right)$ и $f_{\sharp}\left(K_{2}\right)$ гомологичны.

Таким образом, каждый класс когомологий $\psi \in H^{n}\left(\mathscr{T}^{*}(G)\right)$ определяет для любого многообразия $M^{m}$ класс гомологий $\psi_{\sharp}\left(M^{m}\right) \in H_{m-n}\left(M^{m} ; \widehat{G}\right)$ и, следовательно, по двойственности Пуанкаре, класс когомологий $\psi^{\sharp}\left(M^{m}\right) \in H^{n}\left(M^{m} ; G\right)$. Если $m=n$ и многообразие $M^{n}$ ориентированно, то класс $\psi$ определяет элемент группы $G$ по формуле $\psi^{\star}\left(M^{n}\right)=\left\langle\psi^{\sharp}\left(M^{n}\right),\left[M^{n}\right]\right\rangle$. В п. 8 нам потребуется следуюшее следствие предложения 7.2. 
СлЕДСТВИЕ 7.2. Пусть $\psi \in H^{n}\left(\mathscr{T}^{*}(G)\right)$. Тогда $\psi^{\star}\left(M_{1}^{n}\right)=\psi^{\star}\left(M_{2}^{n}\right)$ для любых двух кобордантных ориентированных многообразий $M_{1}^{n}$ и $M_{2}^{n}$.

8. Когомологии комплекса $\mathscr{T}^{*}(\mathbb{Q})$. Характеристическими классами будем называть элементы групы когомологий $H^{*}(\mathrm{BPL} ; G)$, где $\mathrm{BPL}$ - классифицируюшее пространство стабильных кусочно линейных расслоений. Если $p \in H^{*}(\mathrm{BPL} ; G)$, то соответствуюший характеристический класс многообразия $M^{m}$ будем обозначать через $p\left(M^{m}\right)$. В случае $G=\mathbb{Q}$ имеем $H^{*}(\mathrm{BPL} ; \mathbb{Q})=H^{*}(\mathrm{BO} ; \mathbb{Q})$ и характеристические классы - это в точности полиномы от классов Понтрягина.

ОпредЕлЕниЕ 8.1. Локальная формула $f \in \mathscr{T}^{*}(G)$ называется локальной формулой для характеристического класса $p \in H^{*}(\mathrm{BPL} ; G)$, если для любого комбинаторного многообразия $K$ цикл $f_{\sharp}(K)$ представляет класс гомологий, двойственный по Пуанкаре классу когомологий $p(|K|)$.

ТЕОРемА 8.1. Каждая рациональная локальная формула является локальной формулой для некоторого рационального характеристического класса.

ДоКАЗАТЕЛЬСТВО. Из следствия 7.2 вытекает, что корректно определен гомоморфизм $\star: H^{n}\left(\mathscr{T}^{*}(G)\right) \rightarrow \operatorname{Hom}\left(\Omega_{n}, G\right)$, сопоставляюший классу когомологий $\psi$ гомоморфизм $\psi^{\star}$, где $\Omega_{*}$ - кольцо ориентированных кусочно линейных кобордизмов. Сушествует канонический изоморфизм $\operatorname{Hom}\left(\Omega_{n}, \mathbb{Q}\right) \cong H^{n}(\mathrm{BPL} ; \mathbb{Q})$. Поэтому гомоморфизм $\star$ задает гомоморфизм $\sharp: H^{n}\left(\mathscr{T}^{*}(\mathbb{Q})\right) \rightarrow H^{n}(\mathrm{BPL} ; \mathbb{Q})$. Таким образом, для любой локальной формулы $f \in \mathscr{T}^{n}(\mathbb{Q})$ определен рациональньй характеристический класс $p=\sharp(\psi)$, где $\psi$ - класс когомологий, представленньй коциклом $f$. Однако мы еше не доказали, что $f$ - локальная формула для характеристического класса $p$. Действительно, из определения гомоморфизма $\sharp$ следует лишь, что $\psi^{\sharp}\left(M^{n}\right)=p\left(M^{n}\right)$ для любого $n$-мерного многообразия $M^{n}$. Поэтому чтобы доказать, что $f$ - локальная формула для характеристического класса $p$, нам остается доказать следующее предложение.

ПРЕДЛОЖЕНИЕ 8.1. Имеем $\psi^{\sharp}\left(M^{m}\right)=p\left(M^{m}\right)=\sharp(\psi)\left(M^{m}\right)$ для любого многообразия $M^{m}, m \geqslant n$.

СХемА докАЗАТЕльСТвА. Можно показать, что $\left.\psi^{\sharp}\left(M^{m}\right)\right|_{N^{n}}=\psi^{\sharp}\left(N^{n}\right)$ для любого подмногообразия $N^{n} \subset M^{m}$ с тривиальным нормальным расслоением. Если $m>2 n+1$ и многообразие $M^{m}$ ориентируемо, то предложение следует из результата Р. Тома [30] о том, что для любого класса гомологий $z \in H_{n}\left(M^{m} ; \mathbb{Z}\right)$ существует ненулевое целое число $q$ такое, что класс гомологий $q z$ реализуется подмногообразием с тривиальным нормальным расслоением. Если $n<m \leqslant 2 n+1$, нужно заменить многообразие $M^{m}$ на $M^{m} \times S^{n}$, если многообразие $M^{m}$ неориентируемо - перейти к двулистному ориентирующему накрытию.

Первый результат о существовании локальных формул для характеристических классов был получен Н. Левиттом и К. Рурком в [17].

Tеорема 8.2 (Н. Левитт, К. Рурк [17]). Пусть $p \in H^{n}(\mathrm{BPL} ; \mathbb{Q})$ - рациональный характеристический класс, $m \geqslant n-$ челое число. Тогда существует функиия $f \in$ $\mathscr{T}^{n}(\mathbb{Q})$ такая, что для любого ориентированного т-мерного комбинаторного многообразия $K$ класс гомологий, представляемый циклом $f_{\sharp}(K)$, двойствен по Пуанкаре классу когомологий $p(|K|)$. 
ЗАмЕчАНИЕ 8.1. В случае гладкого многообразия с гладкой триангулящией аналогичньй результат был получен П. Л. Кингом [31] (для $m=n$ и вешественных коэффициентов).

В [12] автором доказана следуюшая теорема.

ТЕОРема 8.3. Пусть $p$ - произвольный рациональный характеристический класс. Тогда локальная формула для характеристического класса р существует и единственна с точностью до прибавления произвольной кограниць комплекса $\mathscr{T}^{*}(\mathbb{Q})$. Таким образом, гомоморфизм

$$
\sharp: H^{*}\left(\mathscr{T}^{*}(\mathbb{Q})\right) \rightarrow H^{*}(\mathrm{BPL} ; \mathbb{Q}) \cong \mathbb{Q}\left[p_{1}, p_{2}, \ldots\right], \quad \operatorname{deg} p_{i}=4 i,
$$

является изоморфизмом.

Часть теоремы 8.3, говорящая о сушествовании локальной формулы для произвольного рационального характеристического класса, является усилением теоремы 8.2 и сразу следует из теорем 8.2 и 8.1 .

Н. Левиттом и К. Рурком были также получены результаты о возможности локального вычисления характеристических классов с произвольными коэффициентами для комбинаторных многообразий с заданньм локальным упорядочением вершин, т.е. частичньм упорядочением вершин, ограничение которого на множество вершин звезды каждого симплекса является полньм упорядочением. Пусть $\mathscr{D}_{m}-$ множество ориентированных PL-триангуляций $m$-мерного диска с полным упорядочением вершин с точностью до изоморфизма, сохраняюшего упорядочение вершин.

Теорема 8.4 (Н. Левитт, К. Рурк [17]). Пусть $p \in H^{n}(\mathrm{BPL} ; G)$ - характеристический класс, $m \geqslant n$ - челое число. Тогда существует функция $g: \mathscr{D}_{m} \rightarrow G$ такая, что для любого ориентированного разия $K$ с локальным упорядочением вершин класс гомологий, представляемьй чиклом

$$
\sum_{\sigma^{m-n} \in K} g\left(\operatorname{star} \sigma^{m-n}\right) \sigma^{m-n},
$$

двойствен по Пуанкаре классу когомологий $p(|K|)$.

ЗАмечАнИЕ 8.2. Заметим, что в теореме 8.4 имеются в виду кусочно линейные характеристические классы. В случае $G=\mathbb{Z}$ целочисленные классы Понтрягина не являются кусочно линейными характеристическими классами, но некоторые их кратные являются.

9. Бизвездные преобразования. Пусть $K$ - комбинаторное многообразие. Предположим, что симплициальный комплекс $K$ содержит симплекс $\sigma_{1}$ такой, что $\operatorname{link} \sigma_{1}=\partial \sigma_{2}-$ граница симплекса и симплекс $\sigma_{2}$ не принадлежит комплексу $K$. Тогда $\sigma_{1} * \partial \sigma_{2}-$ полньй подкомплекс комплекса $K$. Бизвездным преобразованием $\beta$, ассоциированным с симплексом $\sigma_{1} \in K$, назьвается преобразование, переводящее комплекс $K$ в симплищиальный комплекс

$$
\beta(K)=\left(K \backslash\left(\sigma_{1} * \partial \sigma_{2}\right)\right) \cup\left(\partial \sigma_{1} * \sigma_{2}\right) .
$$


При этом если $\operatorname{dim} \sigma=0$, считаем, что $\partial \sigma=\varnothing ;$ для любого симплекса $\sigma$ считаем, что $\sigma * \varnothing=\sigma$. Таким образом, частными случаями бизвездных преобразований являются звездные подразделения симплексов максимальной размерности и обратные к ним преобразования. В результате любого бизвездного преобразования получается комбинаторное многообразие, PL-гомеоморфное исходному. На рис. 1 и 2 изображены все виды бизвездных преобразований для случаев $\operatorname{dim} K=2$ и $\operatorname{dim} K=3$.

По теореме Пахнера (см. [32], а также [33]), если $K_{1}$ и $K_{2}$ - две кусочно линейные триангулящии одного многообразия, то $K_{1}$ переводится в $K_{2}$ конечной последовательностью бизвездных преобразований (здесь мы рассматриваем триангуляции как чисто комбинаторные объекты, т.е. мы не различаем изоморфные триангуляции). В частности, любые две $m$-мерные PL-сферы переводятся друг в друга конечной последовательностью бизвездных преобразований.

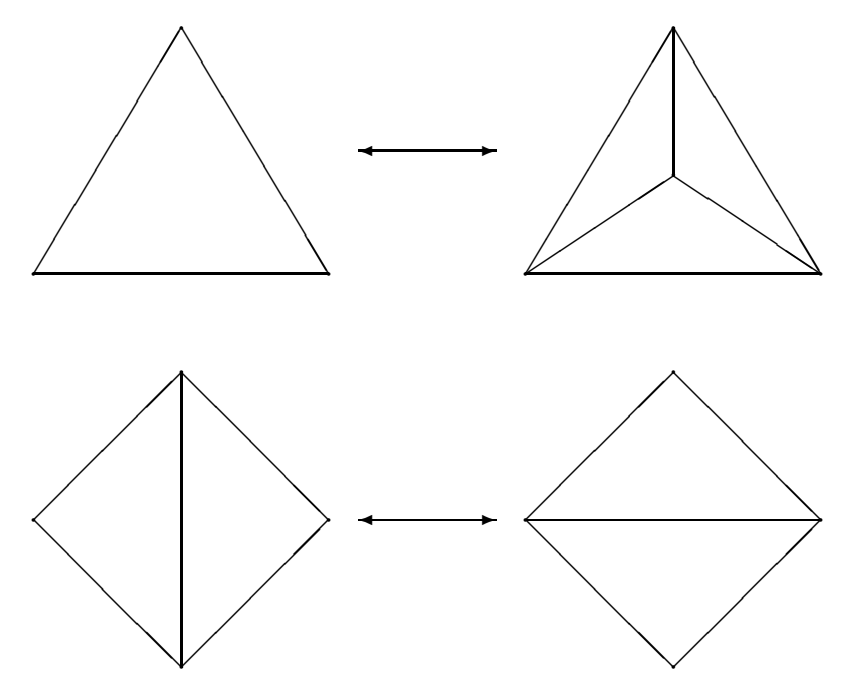

Рис. 1. Бизвездные преобразования для $\operatorname{dim} K=2$

10. Графы $\Gamma_{n}$. Для каждого натурального $n$ определим граф $\Gamma_{n}$. Множество вершин этого графа - множество $\mathscr{T}_{n+1}$ ориентированных $n$-мерных PL-сфер. Пусть $L_{1}, L_{2} \in \mathscr{T}_{n+1}$. Будем говорить, что два бизвездные преобразования $\beta_{1}$ и $\beta_{2}$, переводящие $L_{1}$ в $L_{2}$, ассоциированные с симплексами $\sigma_{1}$ и $\sigma_{2}$ соответственно, әквивалентны, если сушествует автоморфизм PL-сферы $L_{1}$, переводящий симплекс $\sigma_{1}$ в симплекс $\sigma_{2}$. Ребра, соединяюшие две различные вершины $L_{1}$ и $L_{2}$ граф̆а $\Gamma_{n}$, находятся во взаимно однозначном соответствии с классами эквивалентности бизвездных преобразований, переводяших $L_{1}$ в $L_{2}$. Опишем теперь ребра графа $\Gamma_{n}$, оба конца которых совпадают с вершиной $L$. Будем назьвать бизвездное преобразование $\beta$, переводящее PL-cферу $L$ в себя, несущественнылм, если оно эквивалентно обратному бизвездному преобразованию $\beta^{-1}$. Классам эквивалентности несущественных бизвездных преобразований не будет соответствовать никакое ребро графа $\Gamma_{n}$. Остальные классы эквивалентности бизвездных преобразований, переводящих $L$ в себя, разбиваются на 

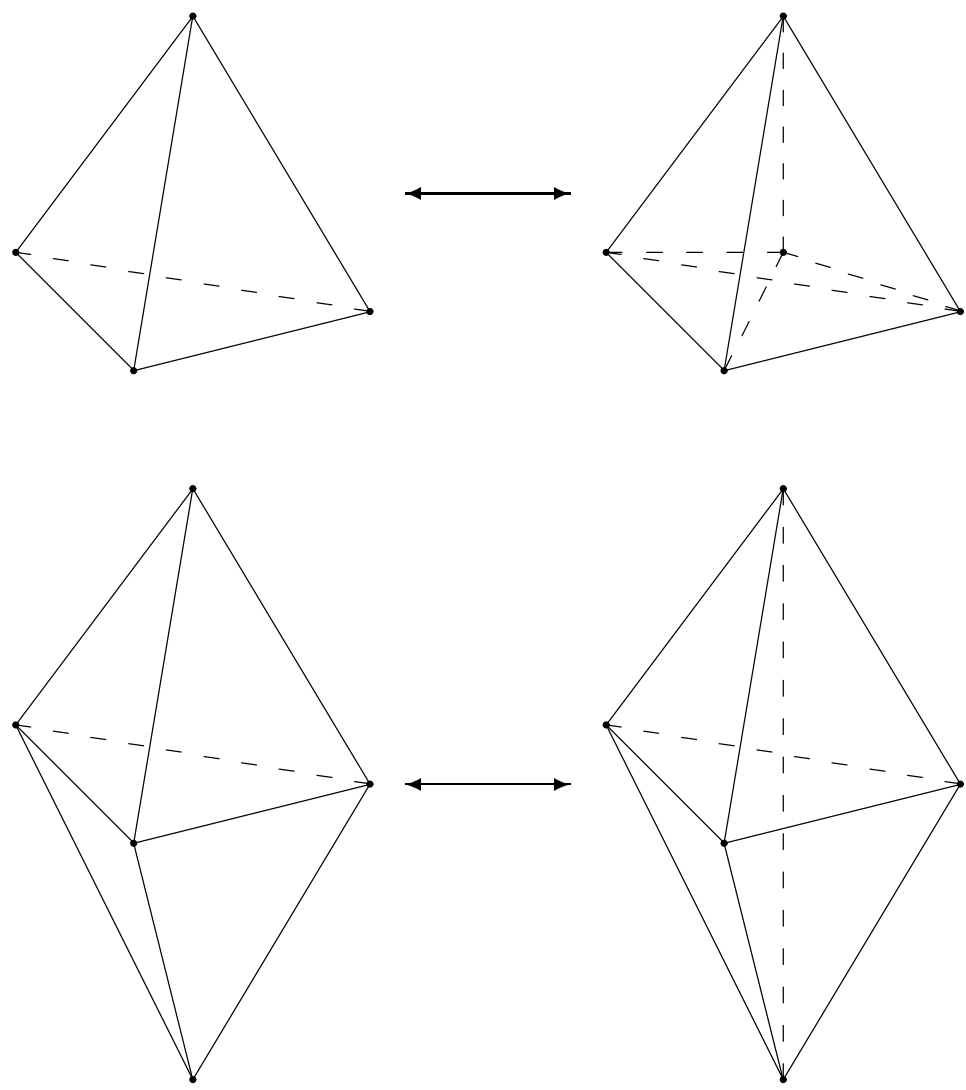

Рис. 2. Бизвездные преобразования для $\operatorname{dim} K=3$

пары взаимно обратных. Ребра графа $\Gamma_{n}$, соединяюшие вершину $L$ с собой, находятся во взаимно однозначном соответствии с такими парами классов эквивалентности. Из теоремы Пахнера следует, что граф̆ $\Gamma_{n}$ связен. Обозначим через $e_{\beta}$ ребро, соответствуюшее бизвездному преобразованию $\beta$. Обозначениям $e_{\beta}$ и $e_{\beta}{ }^{-1}$ будет соответствовать одно и то же ребро, но с разными ориентациями.

Пусть $C_{*}\left(\Gamma_{n} ; \mathbb{Z}\right)$ - клеточный цепной комплекс графа $\Gamma_{n}$. Группа $\mathbb{Z}_{2}$ действует на граффе $\Gamma_{n}$ изменением ориентаций PL-сфер и на групе $\mathbb{Q}$ изменением знака. Следовательно, можно определить эквивариантные коцепи $C_{\mathbb{Z}_{2}}^{*}\left(\Gamma_{n} ; \mathbb{Q}\right)=\operatorname{Hom}_{\mathbb{Z}_{2}}\left(C_{*}\left(\Gamma_{n} ; \mathbb{Z}\right), \mathbb{Q}\right)$ и эквивариантные когомологии $H_{\mathbb{Z}_{2}}^{*}\left(\Gamma_{n} ; \mathbb{Q}\right)$ (считаем, что на группе $\mathbb{Z}$ группа $\mathbb{Z}_{2}$ действует тривиально). Обозначим через $d$ дифференциал комплекса $C_{\mathbb{Z}_{2}}^{*}\left(\Gamma_{n} ; \mathbb{Q}\right)$ и через $B_{\mathbb{Z}_{2}}^{1}\left(\Gamma_{n} ; \mathbb{Q}\right) \subset C_{\mathbb{Z}_{2}}^{1}\left(\Gamma_{n} ; \mathbb{Q}\right)$ подгруппу эквивариантных кограниц. Из того, что граф̆ $\Gamma_{n}$ связен, следует, что $H_{\mathbb{Z}_{2}}^{0}\left(\Gamma_{n} ; \mathbb{Q}\right)=0$. Поэтому гомоморфизм

$$
d: C_{\mathbb{Z}_{2}}^{0}\left(\Gamma_{n} ; \mathbb{Q}\right) \rightarrow C_{\mathbb{Z}_{2}}^{1}\left(\Gamma_{n} ; \mathbb{Q}\right)
$$

является мономорфизмом.

Очевидно, что $C_{\mathbb{Z}_{2}}^{0}\left(\Gamma_{n-1} ; \mathbb{Q}\right)=\mathscr{T}^{n}(\mathbb{Q})$. Поэтому определен дифференциал

$$
\delta: C_{\mathbb{Z}_{2}}^{0}\left(\Gamma_{n-1} ; \mathbb{Q}\right) \rightarrow C_{\mathbb{Z}_{2}}^{0}\left(\Gamma_{n} ; \mathbb{Q}\right) .
$$


Пусть $L_{1}, L_{2} \in \mathscr{T}_{n+1}, \beta$ - бизвездное преобразование, переводящее $L_{1}$ в $L_{2}$. Можно считать, что $L_{1}$ и $L_{2}-$ симплициальные комплексы с одним и тем же множеством вершин $V$ (если $\operatorname{dim} \sigma_{1}>0$ и $\operatorname{dim} \sigma_{2}>0$, то это действительно так, иначе введем для одного из комплексов $L_{1}$ и $L_{2}$ фиктивную вершину $v_{0}$, которая не будет симплексом этого комплекса). Для любой вершины $v \in V$ бизвездное преобразование $\beta$ либо не изменяет комплекс link $v$, либо индуцирует бизвездное преобразование $\beta_{v}$, переводящее комплекс $\operatorname{link}_{L_{1}} v$ в комплекс $\operatorname{link}_{L_{2}} v$. Обозначим через $W \subset V$ подмножество, состояшее из всех вершин $v$ таких, что бизвездное преобразование $\beta_{v}$ не является несушественным (считаем, что $\left.v_{0} \notin W\right)$. Определим дифференциал $\delta: C_{\mathbb{Z}_{2}}^{1}\left(\Gamma_{n-1} ; \mathbb{Q}\right) \rightarrow C_{\mathbb{Z}_{2}}^{1}\left(\Gamma_{n} ; \mathbb{Q}\right)$ по формуле

$$
(\delta h)\left(e_{\beta}\right)=\sum_{v \in W} h\left(e_{\beta_{v}}\right)
$$

Легко проверить, что $\delta^{2}=0$ и $\delta d=d \delta$.

11. Локальные формулы для первого класса Понтрягина. Этот пункт посвящен явному описанию всех локальных формул для первого класса Понтрягина. По теореме 8.1 каждая локальная формула $f \in \mathscr{T}^{4}(\mathbb{Q})$ является локальной формулой для первого класса Понтрягина, умноженного на некоторое рациональное число. Таким образом, прежде всего нужно научиться находить все функции $f: \mathscr{T}_{4} \rightarrow \mathbb{Q}$, являюшиеся локальньми формулами. Любую функцию $f \in \mathscr{T}^{4}(\mathbb{Q})$ можно рассматривать как $\mathbb{Z}_{2}$-эквивариантную нульмерную клеточную коцепь на графе $\Gamma_{3}$.

ПРЕДЛОЖЕНИЕ 11.1. Функиия $f \in \mathscr{T}^{4}(\mathbb{Q})=C_{\mathbb{Z}_{2}}^{0}\left(\Gamma_{3} ; \mathbb{Q}\right)$ является локальной формулой тогда и только тогда, когда существует коцепь $h \in C_{\mathbb{Z}_{2}}^{1}\left(\Gamma_{2} ; \mathbb{Q}\right)$ такая, что $d f=\delta h$.

ДокаЗАтельство. Пусть существует коцепь $h \in C_{\mathbb{Z}_{2}}^{1}\left(\Gamma_{2} ; \mathbb{Q}\right)$ такая, что $d f=\delta h$. Тогда $d \delta f=\delta d f=\delta^{2} h=0$. Следовательно, $\delta f=0$, так как $d: C_{\mathbb{Z}_{2}}^{0}\left(\Gamma_{4} ; \mathbb{Q}\right) \rightarrow$ $C_{\mathbb{Z}_{2}}^{1}\left(\Gamma_{4} ; \mathbb{Q}\right)$ - мономорфизм. Значит, $f$ - локальная формула.

Пусть теперь известно, что $f$ - локальная формула.

Пусть $L_{1}, L_{2} \in \mathscr{T}_{n}, \beta$ - бизвездное преобразование, переводящее PL-сферу $L_{1}$ в PL-cферу $L_{2}$. Определим множества $V$ и $W$ так же, как в п. 10 . Пусть $\beta$ заменяет полный подкомплекс $\sigma_{1} * \partial \sigma_{2}$ симплициального комплекса $L_{1}$ на полньй подкомплекс $\partial \sigma_{1} * \sigma_{2}$ симплициального комплекса $L_{2}$. Рассмотрим конус $C L_{1}$ с вершиной $u_{1}$ и конус $C L_{2}$ с вершиной $u_{2}$. Тогда $L_{\beta}=C L_{1} \cup C L_{2} \cup\left(\sigma_{1} * \sigma_{2}\right)$ - симплициальньй комплекс на множестве вершин $V \cup\left\{u_{1}, u_{2}\right\}$. Очевидно, что $L_{\beta}$ является $n$-мерной PL-сферой. Ориентируем $L_{\beta}$ так, чтобы индуцированная ориентация комплекса link $u_{2}=L_{2}$ coвпадала с заданной. Тогда $L_{\beta} \in \mathscr{T}_{n+1}$.

Рассмотрим коцепь $h \in C_{\mathbb{Z}_{2}}^{1}\left(\Gamma_{2} ; \mathbb{Q}\right)$ такую, что $h\left(e_{\beta}\right)=f\left(L_{\beta}\right)$ для любого ребра $e_{\beta}$ граффа $\Gamma_{2}$. Докажем, что $\delta h=d f$. Пусть $e_{\beta}$ - произвольное ребро графа $\Gamma_{3}$, где $\beta-$ бизвездное преобразование, переводящее трехмерную PL-сферу $L_{1}$ в PL-сферу $L_{2}$. Очевидно, что линки всех вершин $v \in V \backslash W$ в комплексе $L_{\beta}$ симметричны. Линк вершины $v \in W$ в комплексе $L_{\beta}$ изоморфен комплексу $-L_{\beta_{v}}$. Линки вершин $u_{1}$ и $u_{2}$ изоморфны соответственно комплексам $-L_{1}$ и $L_{2}$. Из того, что $f$ - локальная формула, следует, 
что

$$
\begin{aligned}
0=(\delta f)\left(L_{\beta}\right) & =-\sum_{v \in W} f\left(L_{\beta_{v}}\right)+f\left(L_{2}\right)-f\left(L_{1}\right) \\
& =-\sum_{v \in W} h\left(e_{\beta_{v}}\right)+f\left(\partial e_{\beta}\right)=-(\delta h)\left(e_{\beta}\right)+(d f)\left(e_{\beta}\right) .
\end{aligned}
$$

Предложение 11.1 доказано.

Опишем теперь подгруппу $A \subset C_{\mathbb{Z}_{2}}^{1}\left(\Gamma_{2} ; \mathbb{Q}\right)$, состоящую из всех коцепей $h$ таких, что $\delta h \in B_{\mathbb{Z}_{2}}^{1}\left(\Gamma_{3} ; \mathbb{Q}\right)$, т.е. $\delta h=d f$ для некоторой коцепи $f \in C_{\mathbb{Z}_{2}}^{0}\left(\Gamma_{3} ; \mathbb{Q}\right)$. Из того, что дифференциалы $d$ и $\delta$ коммутируют, следует, что $\delta: C_{\mathbb{Z}_{2}}^{*}\left(\Gamma_{2} ; \mathbb{Q}\right) \rightarrow C_{\mathbb{Z}_{2}}^{*}\left(\Gamma_{3} ; \mathbb{Q}\right)$ - цепное отображение. Значит, корректно определен индуцированньй гомоморфизм $\delta^{*}: H_{\mathbb{Z}_{2}}^{1}\left(\Gamma_{2} ; \mathbb{Q}\right) \rightarrow H_{\mathbb{Z}_{2}}^{1}\left(\Gamma_{3} ; \mathbb{Q}\right)$. Обозначим через $\widetilde{A}$ ядро этого гомоморфизма. Пусть $h \in C_{\mathbb{Z}_{2}}^{1}\left(\Gamma_{2} ; \mathbb{Q}\right)$. Очевидно, что $h \in A$ тогда и только тогда, когда $[h] \in \widetilde{A}$.

Очевидно, что $H_{\mathbb{Z}_{2}}^{1}\left(\Gamma_{2} ; \mathbb{Q}\right) \cong \operatorname{Hom}_{\mathbb{Z}_{2}}\left(H_{1}\left(\Gamma_{2} ; \mathbb{Z}\right), \mathbb{Q}\right)$. Поэтому для описания подгрупшы $\widetilde{A}$ нам нужно выбрать систему образуюших в групше $H_{1}\left(\Gamma_{2} ; \mathbb{Z}\right)$. Рассмотрим циклы в графе $\Gamma_{2}$, изображенные на рис. 3-8. Эти рисунки нужно понимать следующим образом: рассматривается произвольная двумерная PL-сфера, содержащая подкомплекс, изображенный на рисунке, и с ней производятся бизвездные преобразования, изображенные на рисунке. При этом предполагается, что ориентация этой двумерной PL-сферы задается обходом по часовой стрелке вершин треугольников, изображенных на рисунке. Если на рисунке некоторый угол выделен дугой, то предполагается, что к данной вершине внутри данного угла примыкает ровно столько треугольников, сколько написано. Таким образом, получаем 6 бесконечных серий циклов в графе $\Gamma_{2}$. Заметим, что циклы, изображенные на рис. $3-5$, соответствуют коммутированию двух бизвездных преобразований.

ПРЕДЛОЖЕНИЕ 11.2. Классь гомологий, задаваемые ииклами, изображсеньми на рис. $3-8$, порождапют группу $H_{1}\left(\Gamma_{2} ; \mathbb{Z}\right)$.

В этой статье мы опускаем доказательство предложения 11.2. Оно опирается на два утверждения, доказанные в [34] (см. также [29]):

1) каждая триангуляция двумерной сфферы может быть реализована в виде границы вьпуклого симплициального многогранника;

2) два комбинаторно эквивалентных трехмерных выпуклых симплициальных многогранника могут быть продеформированы друг в друга в классе вьпуклых симплициальных многогранников с тем же комбинаторным типом.

Обозначим через $S$ множество циклов, изображенных на рис. $3-8$. Определим функцию $c: S \rightarrow \mathbb{Q}$, сопоставив каждому циклу число, написанное на соответствующем рисунке, где

$$
\begin{gathered}
\rho(p, q)=\frac{q-p}{(p+q+2)(p+q+3)(p+q+4)}, \\
\eta(p)=\frac{1}{(p+2)(p+3)} .
\end{gathered}
$$


ЗАмечАниЕ 11.1. Похожие числовые выражения возникали в статье [35] при решении совсем другой задачи, а именно при нахождении формулы для класса Чженя-Эйлера $S^{1}$-расслоения через особенности ограничений морсовской функции на тотальном пространстве на слои этого расслоения.

ПреДлОЖЕнИЕ 11.3. Функиия с продолжается до корректно определенного класса когомологий $c \in H_{\mathbb{Z}_{2}}^{1}\left(\Gamma_{2} ; \mathbb{Q}\right)=\operatorname{Hom}_{\mathbb{Z}_{2}}\left(H_{1}\left(\Gamma_{2} ; \mathbb{Z}\right), \mathbb{Q}\right)$. При этом $\widetilde{A}-$ одномерное векторное пространство, натянутое на класс когомологий $c$.

План доказательства этого предложения будет дан в п. 13.

Из предложения 11.1 следует, что корректно определен гомоморфизм $d^{-1} \delta: A \rightarrow$ $C_{\mathbb{Z}_{2}}^{0}\left(\Gamma_{3} ; \mathbb{Q}\right)=\mathscr{T}^{4}(\mathbb{Q})$, образом которого является подгрупа, состоящая из всех локальных формул.

Tеорема 11.1. Отображение $d^{-1} \delta$ осуществляет биекиию между множеством всех $\mathbb{Z}_{2}$-эквивариантных коциклов, представляющих класс когомологий $c$, и множеством всех локальных формул для первого класса Понтрягина.

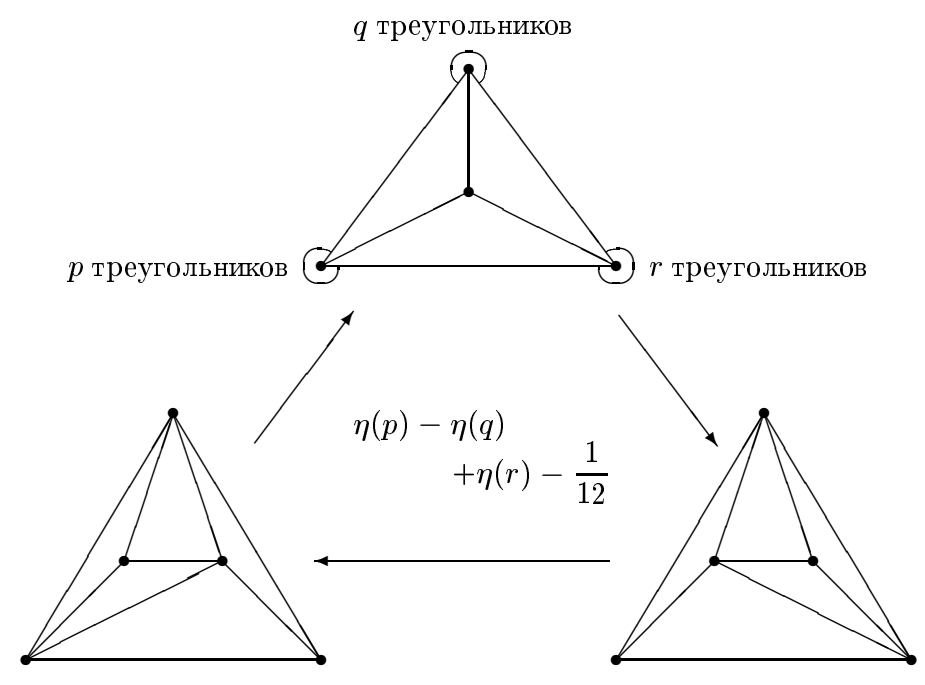

Рис. 6.

ДоказАтЕльство. Рассмотрим эпиморфизм $j: \widetilde{A} \rightarrow H^{4}\left(\mathscr{T}^{*}(\mathbb{Q})\right)$, индуцированньй гомоморфизмом $d^{-1} \delta$. Имеем $\operatorname{dim} \widetilde{A}=1$ по предложению 11.3 и $\operatorname{dim} H^{4}\left(\mathscr{T}^{*}(\mathbb{Q})\right)=$ 1 по теореме 8.3. Значит, эпиморфизм $j$ является изоморфизмом и существует рациональное число $\lambda \neq 0$ такое, что $j(\lambda c)=\varphi$, где $\varphi \in H^{4}\left(\mathscr{T}^{*}(\mathbb{Q})\right)$ - класс когомологий, представляемый локальньми формулами для первого класса Понтрягина.

Докажем, что $d^{-1} \delta$ - мономорфизм. Имеем $\operatorname{ker}\left(d^{-1} \delta\right) \subset B_{\mathbb{Z}_{2}}^{1}\left(\Gamma_{2} ; \mathbb{Q}\right)$, так как $j-$ мономорфизм. При этом $\left.\left(d^{-1} \delta\right)\right|_{B_{\mathbb{Z}_{2}}^{1}\left(\Gamma_{2} ; \mathbb{Q}\right)}=\delta d^{-1}$. Очевидно, что $\mathscr{T}^{2}(\mathbb{Q})=0$. Кроме того по теореме $8.3 H^{3}\left(\mathscr{T}^{*}(\mathbb{Q})\right)=0$. Следовательно, $\delta: \mathscr{T}^{3}(\mathbb{Q}) \rightarrow \mathscr{T}^{4}(\mathbb{Q})$ - мономорфизм. Значит, $\delta d^{-1}: B_{\mathbb{Z}_{2}}^{1}\left(\Gamma_{2} ; \mathbb{Q}\right) \rightarrow \mathscr{T}^{4}(\mathbb{Q})$ - мономорфизм. 

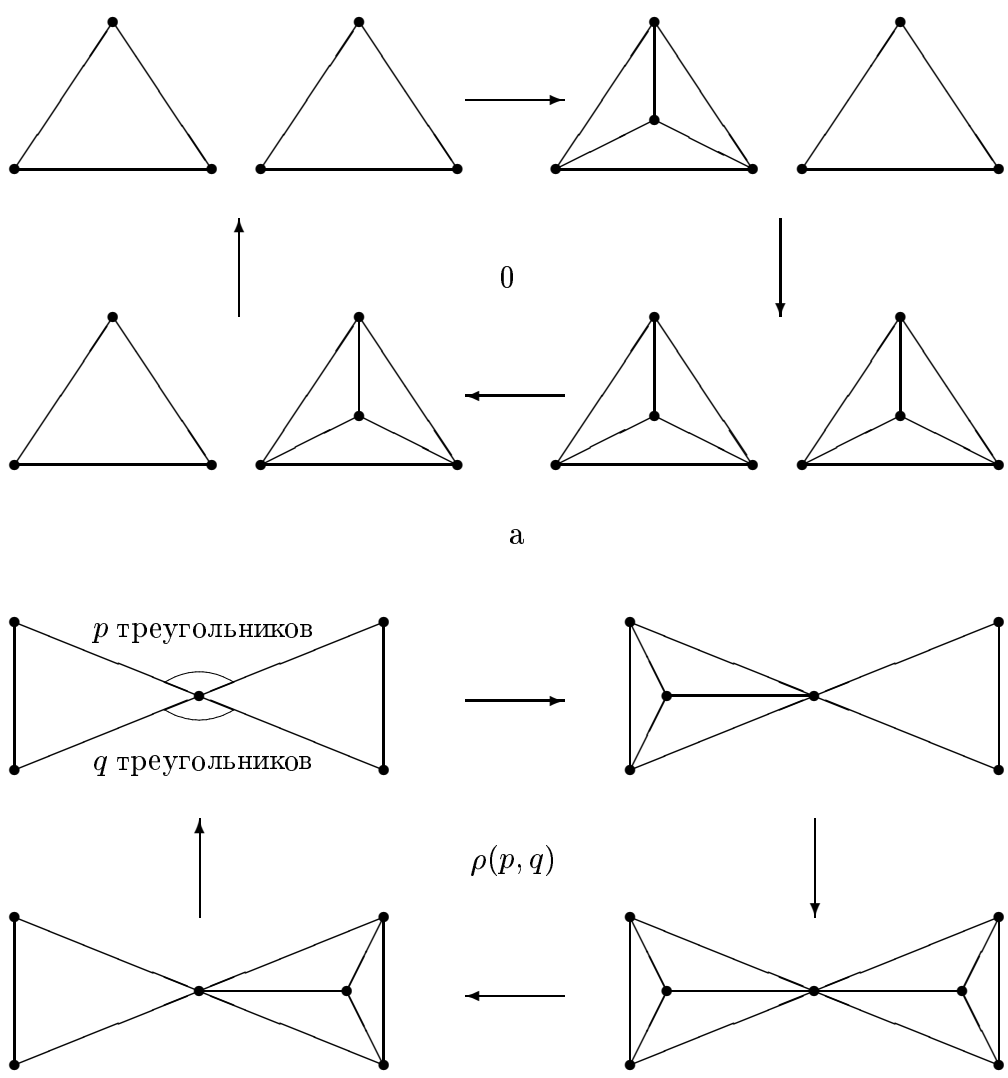

6
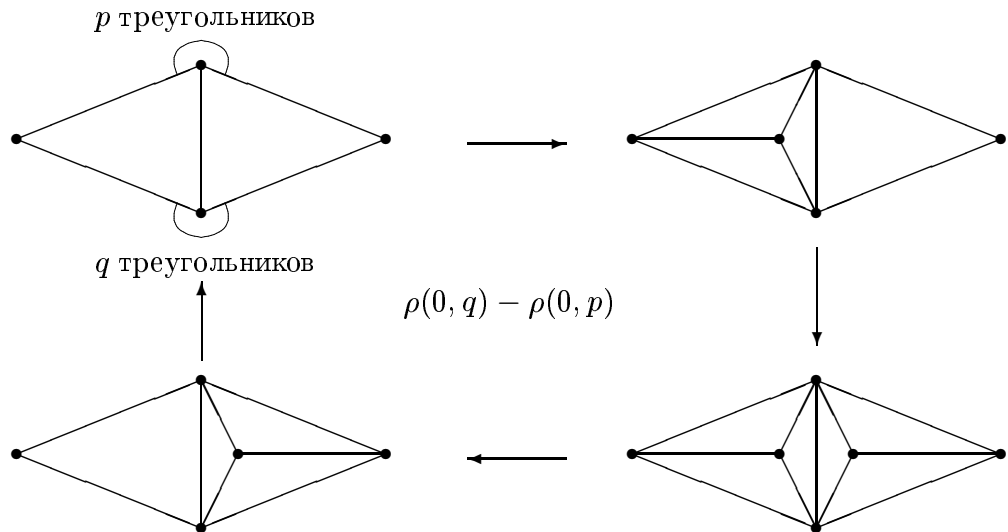

$\rho(0, q)-\rho(0, p)$
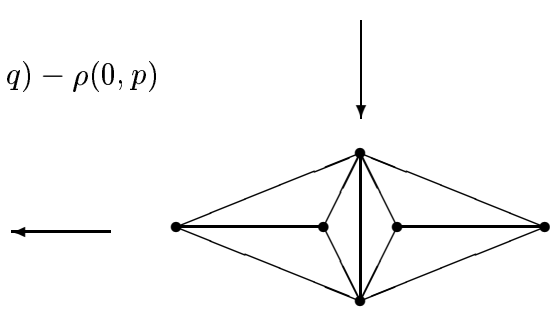

Рис. 3. 

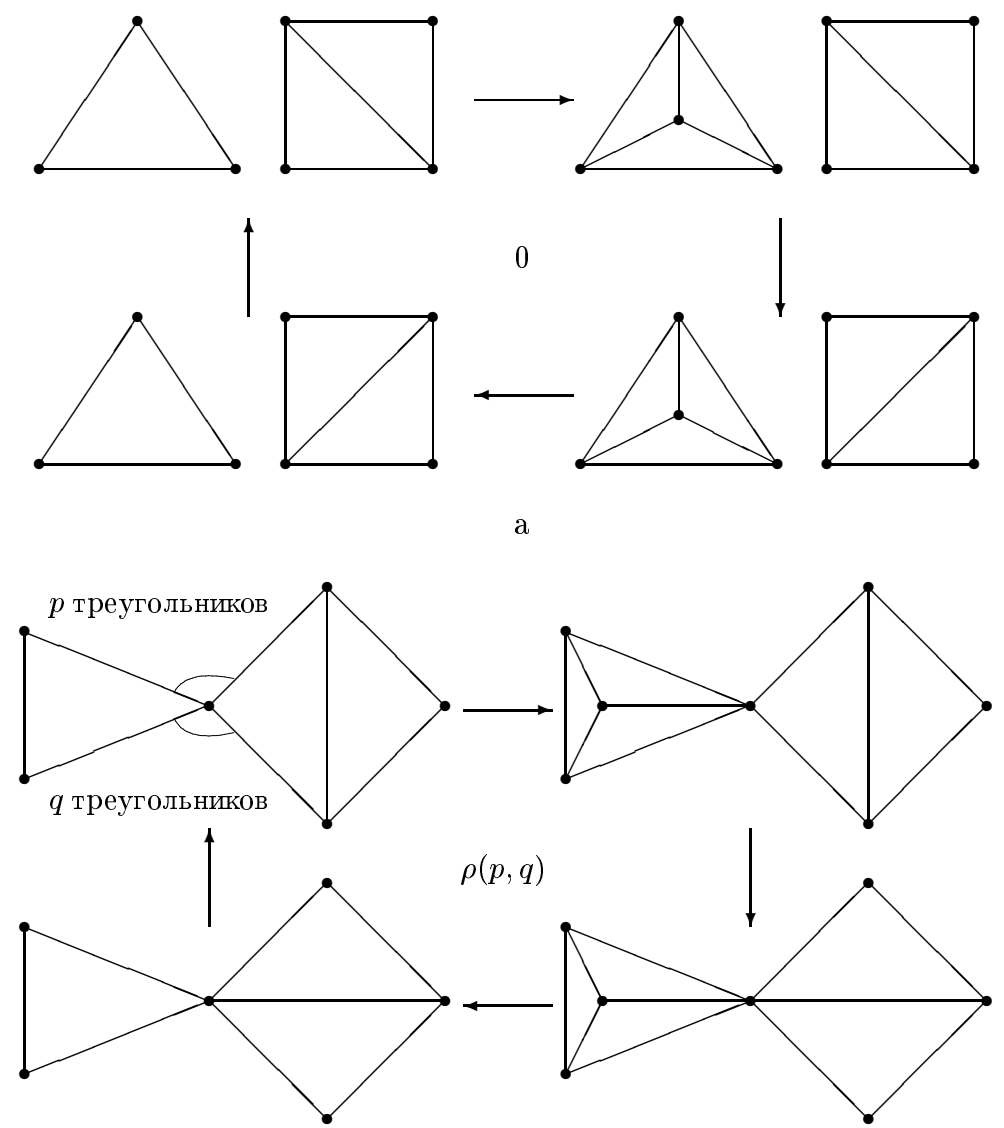

б
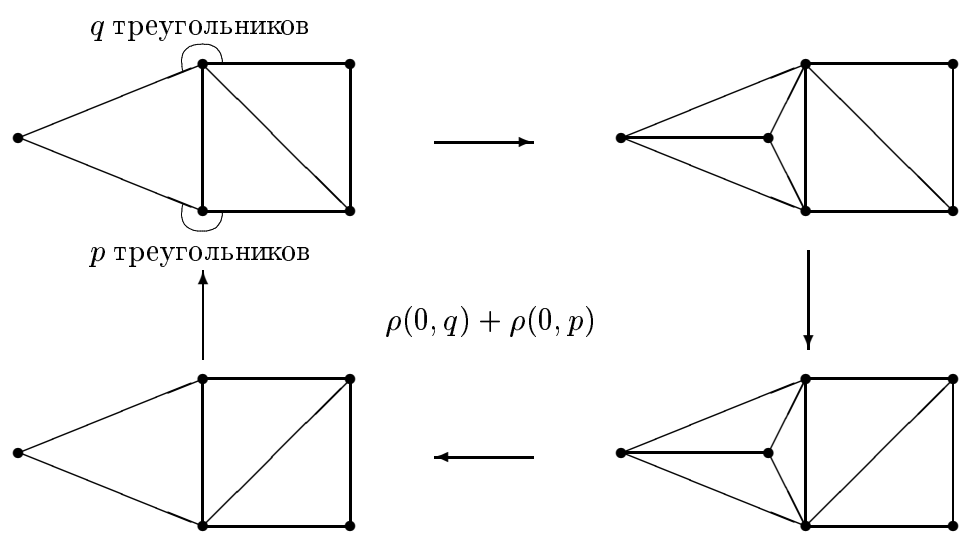

Рис. 4. 

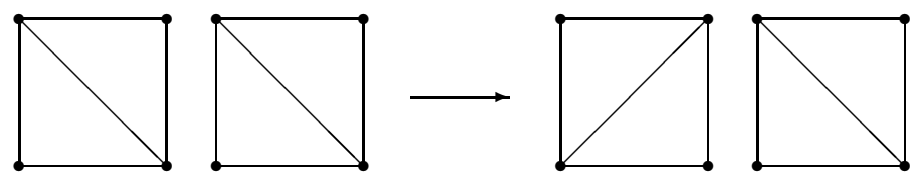

0
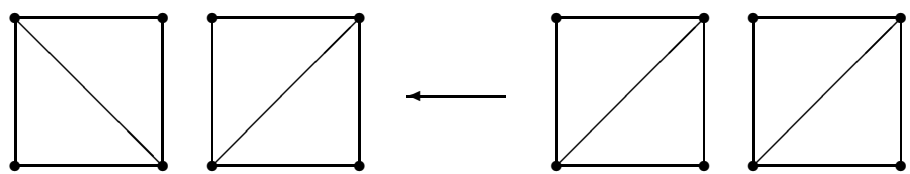

a
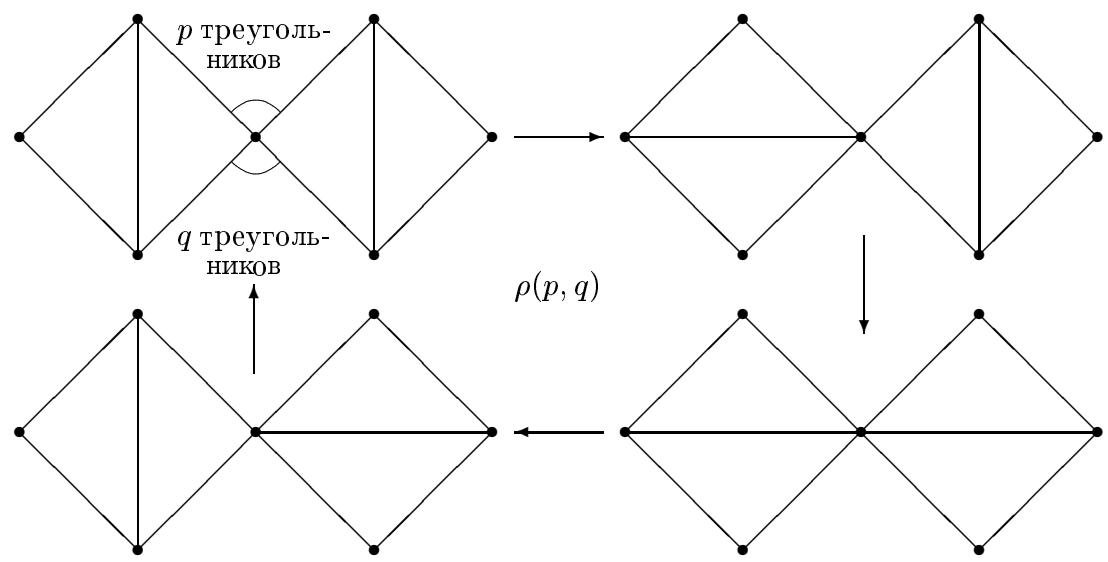

б
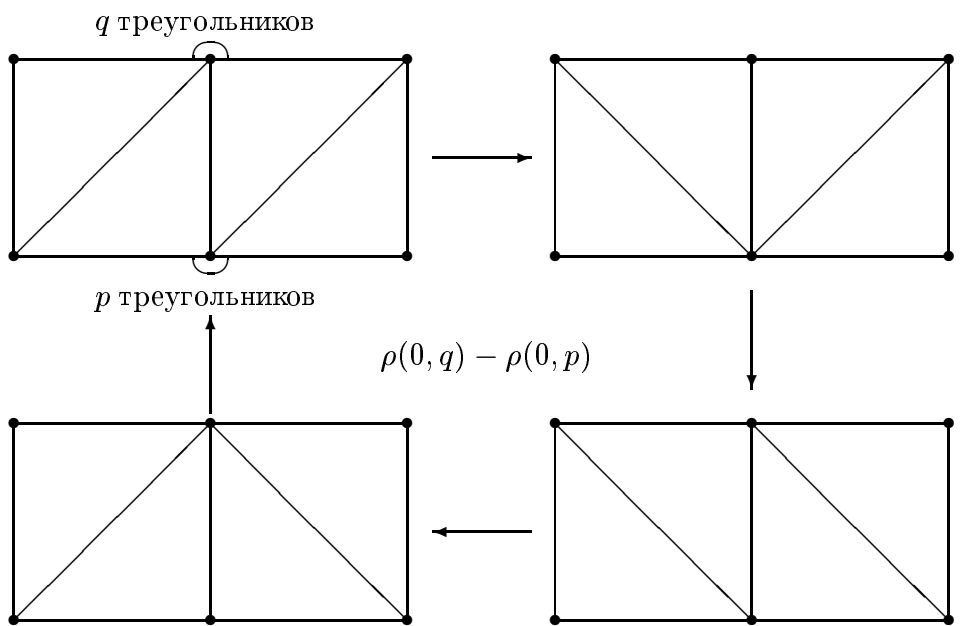

$\rho(0, q)-\rho(0, p)$

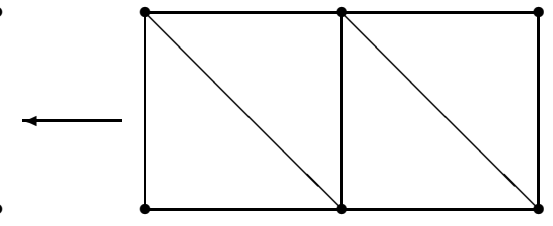

B

Рис. 5. 


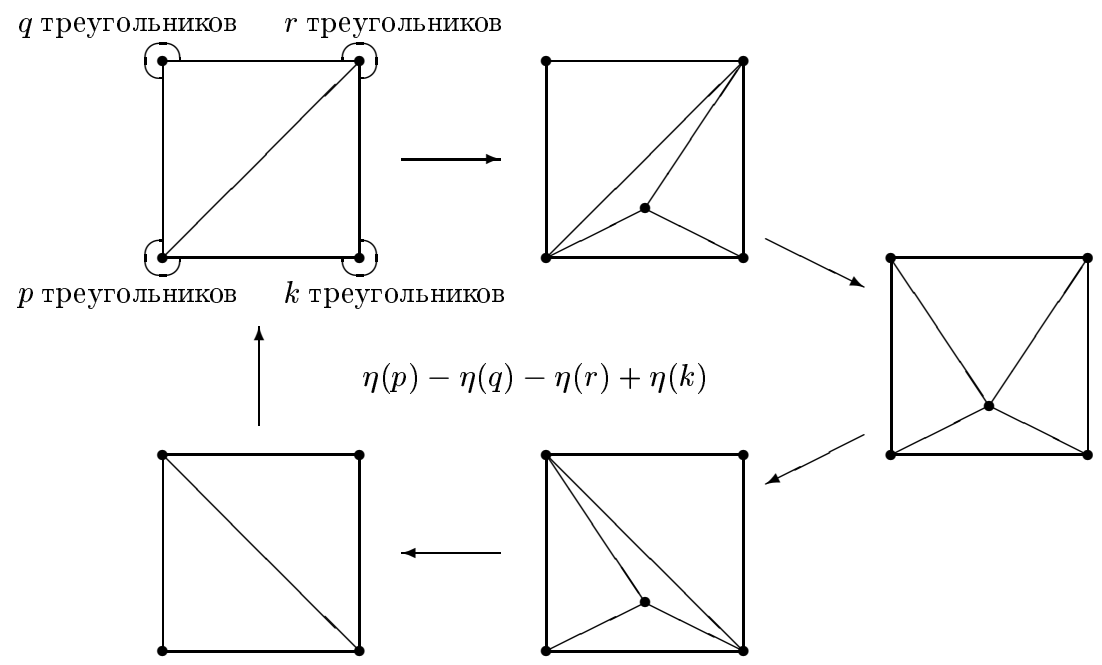

Рис. 7.

Итак, $d^{-1} \delta-$ мономорфизм. Значит, гомоморфизм $d^{-1} \delta$ осушествляет биекцию между множеством всех $\mathbb{Z}_{2}$-эквивариантных коциклов, представляюших класс когомологий $\lambda c$, и множеством всех локальных формул для первого класса Понтрягина. Осталось доказать, что $\lambda=1$. Для этого достаточно рассмотреть какое-нибудь ориентированное четырехмерное комбинаторное многообразие $K$ с известньм первьм числом Понтрягина и вычислить $\psi^{\star}(|K|)$, где $\psi=j(c)$. Выбирая в качестве $K$ триангулящию $\mathbb{C} P^{2}$ с 9 вершинами, построенную в [36] (см. также [37]), с помошю непосредственного вычисления можно проверить, что $\lambda=1$.

ЗАмЕчАНИЕ 11.2. На самом деле при доказательстве этой теоремы используется только та часть теоремы 8.3, которая говорит об эпиморфности отображения $\sharp: H^{*}\left(\mathscr{T}^{*}(\mathbb{Q})\right) \rightarrow H^{*}(\mathrm{BPL} ; \mathbb{Q})\left(\right.$ равенство $H^{3}\left(\mathscr{T}^{*}(\mathbb{Q})\right)=0$ легко проверяется непосредственно).

12. Выбор канонической формулы. Теорема 11.1 дает описание всех рациональных локальных формул для первого класса Понтрягина. Полезно теперь дать описание какой-нибудь одной канонической локальной формулы $f_{0}$ для первого класса Понтрягина, т.е. найти канонический коцикл $\widehat{c}_{0} \in C_{\mathbb{Z}_{2}}^{1}\left(\Gamma_{2} ; \mathbb{Q}\right)$, представляюший класс когомологий $c$. Задача о выборе канонического 1-мерного коцикла, представляющего заданный класс когомологий, возникала (совсем в другой ситуации) при построении формулы работы [9]. Здесь мы пользуемся методом, аналогичньм примененному в этой работе.

Обозначим через $\mathscr{T}_{3}^{(l)}$ множество классов изоморфизма ориентированных 2-мерных PL-cфер, которые могут быть получены из границы тетраэдра с помощью не более чем $l$ бизвездных преобразований. Обозначим через $\Gamma_{2}^{(l)}$ полный подграф графа $\Gamma_{2}$, натянутьй на множество вершин $\mathscr{T}_{3}^{(l)}$. Тогда $\Gamma_{2}^{(l)}-$ конечньй связньй граф, допускающий явное комбинаторное построение. Будем последовательно строить коциклы 


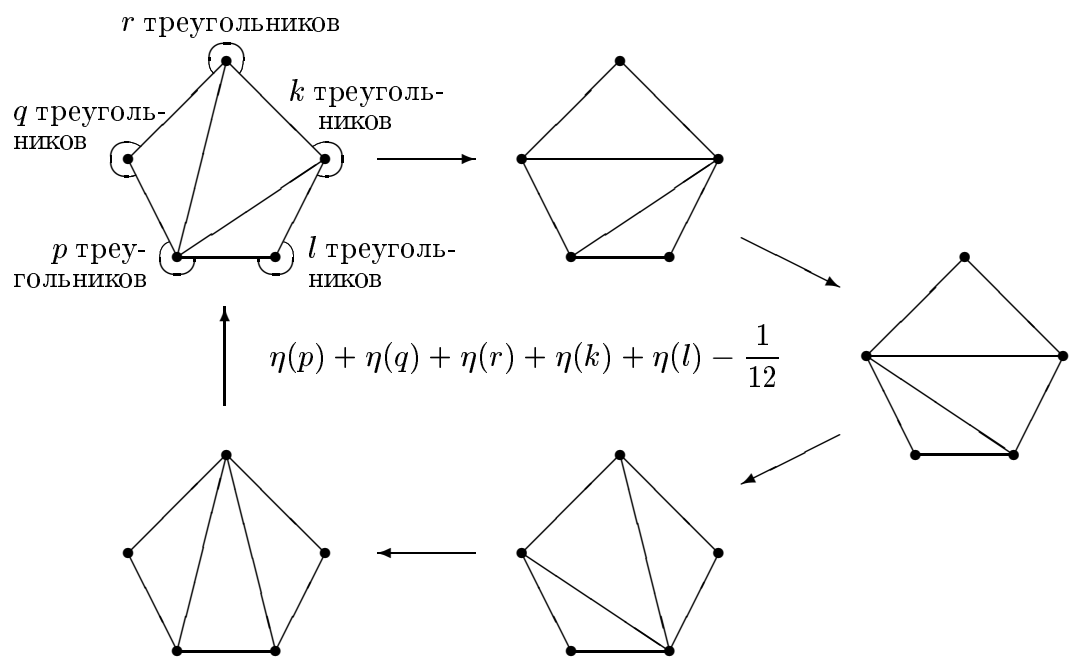

Рис. 8.

$\widehat{c}_{0}^{(l)} \in C_{\mathbb{Z}_{2}}^{1}\left(\Gamma_{2}^{(l)} ; \mathbb{Q}\right)$, представляюшие классы когомологий $\left.c\right|_{\Gamma_{2}^{(l)}}$, такие, что ограничение коцикла $\widehat{c}_{0}^{(l)}$ на граф $\Gamma_{2}^{(l-1)}$ совпадает с коциклом $\widehat{c}_{0}^{(l-1)}$. Пусть коцикл $\widehat{c}_{0}^{(l-1)}$ уже построен. Из всех коциклов $b \in C_{\mathbb{Z}_{2}}^{1}\left(\Gamma_{2}^{(l)} ; \mathbb{Q}\right)$ таких, что $[b]=\left.c\right|_{\Gamma_{2}^{(l)}}$ и $\left.b\right|_{\Gamma_{2}^{(l-1)}}=\widehat{c}_{0}^{(l-1)}$, в качестве $\widehat{c}_{0}^{(l)}$ выберем тот, для которого сумма квадратов его значений на всех ребрах графа $\Gamma_{2}^{(l)}$ достигает наименьшего значения. Задача о выборе такого коцикла является задачей о минимизации квадратичного функционала на плоскости в конечномерном векторном пространстве. Поэтому искомьй коцикл существует, единственен, рационален и его выгисление сводится к решению системы линейных уравнений с рациональными коэффициентами. Обозначим через $\widehat{c}_{0}$ коцикл на графе $\Gamma_{2}$, ограничения которого на графы $\Gamma_{2}^{(l)}$ совпадают с коциклами $\widehat{c}_{0}^{(l)}$. Тогда $f_{0}=d^{-1} \delta \widehat{c}_{0}$-каноническая локальная фоормула для первого класса Понтрягина.

Опишем, как производить конкретные вычисления с помошю этой формулы, т.е. как по заданной PL-сфере $L \in \mathscr{T}_{4}$ вычислить значение $f_{0}(L)$. Для этого нужно произвести следующую последовательность действий.

1) Выберем последовательность бизвездных преобразований $\beta_{1}, \ldots, \beta_{l}$, переводящих границу 4 -мерного симплекса в PL-сферу $L$. Обозначим через $L_{j}$ PL-сферу, полученную из $\partial \Delta^{4}$ применением бизвездных преобразований $\beta_{1}, \ldots, \beta_{j-1}$, и через $W_{j}-$ множество вершин $v \in L_{j}$ таких, что бизвездное преобразование $\beta_{j}$ индуцирует в линке вершины $v$ бизвездное преобразование $\beta_{j}$, не являюшееся несушественным. Заметим, что все ребра $e_{\beta_{j v}}$ лежат в графе $\Gamma_{2}^{(l)}$.

2 ) Последовательно вычислим графы $\Gamma_{2}^{(j)}$ и коциклы $\widehat{c}_{0}^{(j)}, j=1, \ldots, l$.

3) Вычислим значение $f_{0}(L)$ по формуле

$$
f_{0}(L)=\sum_{j=1}^{l} \sum_{v \in W_{j}} \widehat{c}_{0}^{(l)}\left(e_{\beta_{j v}}\right) .
$$


13. План доказательства предложения 11.3. Пусть $c^{\prime}$ - произвольный элемент группы $\widetilde{A}$. Докажем сначала, что существует $\lambda \in \mathbb{Q}$ такое, что $c^{\prime}([\alpha])=\lambda c([\alpha])$ для любого $\alpha \in S$.

Пусть $\alpha$ - цикл, изображенный на рис. 3a, $L$ - исходная двумерная PL-сфера (PL-cфера, изображенная в левом верхнем углу рис. $3 \mathrm{a}), \sigma_{1}$ и $\sigma_{2}-$ треугольники PL-cферы $L$, изображенные на рисунке. Рассмотрим трехмерную PL-сферу $K$, содержашую вершину $u$, звезда которой является полным подкомплексом комплекса $K$, а линк изоморфен PL-сфере $L$. Обозначим через $\widetilde{\sigma}_{1}$ и $\widetilde{\sigma}_{2}$ тетраэдры PL-сферы $K$, натяну тые на вершину $u$ и треугольники $\sigma_{1}$ и $\sigma_{2}$ соответственно. Цикл $\alpha$ получался при коммутировании бизвездных преобразований, ассоциированных с треугольниками $\sigma_{1}$ и $\sigma_{2}$. Прокоммутируем теперь бизвездные преобразования, ассоциированные с тетраэдрами $\widetilde{\sigma}_{1}$ и $\widetilde{\sigma}_{2}$. Получим цикл $\gamma \in C_{1}\left(\Gamma_{3} ; \mathbb{Z}\right)$. Цикл $\gamma$ индуцирует для каждой вершины $v \in K$ цикл $\gamma_{v}$ в графе $\Gamma_{2}$, состояший из ребер, соответствующих индуцированным бизвездньм преобразованиям линка вершины $v$. Тогда

$$
\sum_{v} c^{\prime}\left(\left[\gamma_{v}\right]\right)=\delta^{*}\left(c^{\prime}\right)([\gamma])=0
$$

так как $c^{\prime} \in \widetilde{A}=\operatorname{ker} \delta^{*}$. Для всех вершин $v$ комплекса $K$, кроме вершины $u$, циклы $\gamma_{v}$ гомологичны нулю. Цикл $\gamma_{u}$ совпадает с циклом $\alpha$. Значит, $c^{\prime}([\alpha])=0$.

Пусть теперь $\alpha_{1}$ и $\alpha_{2}-$ два цикла, изображенные на рис. 3б с одинаковыми парами чисел $(p, q)$. Аналогично предыдушему случаю, можно найти цикл $\gamma \in C_{1}\left(\Gamma_{3} ; \mathbb{Z}\right)$, начинающийся с некоторой трехмерной PL-сферы $K$ такой, что $\gamma_{u}=\alpha_{1}, \gamma_{v}=-\alpha_{2}$ для некоторых вершин $u, v$ комплекса $K$ и $\gamma_{w}=0$ для всех остальных вершин $w$ комплекса $K$. Значит, $c^{\prime}\left(\left[\alpha_{1}\right]\right)=c^{\prime}\left(\left[\alpha_{2}\right]\right)$. Таким образом, значение функции $c^{\prime}$ на цикле, изображенном на рис. $3 б$, зависит только от пары чисел $(p, q)$. Обозначим это значение через $\rho^{\prime}(p, q)$. Аналогичными рассуждениями устанавливается, что функцию $\rho^{\prime}(p, q)$ можно доопределить до функции $\mathbb{Z}_{\geqslant 0} \times \mathbb{Z}_{\geqslant 0} \rightarrow \mathbb{Q}$ такой, что $\rho^{\prime}(p, q)=-\rho^{\prime}(q, p)$ и $c^{\prime}([\alpha])=\rho^{\prime}(0, q)-\rho^{\prime}(0, p)$ для любого цикла $\alpha$, изображенного на рис. Зв. Такое доопределение можно произвести многими способами. Чтобы фиксировать один способ, предположим, что $\rho^{\prime}(0,1)=\frac{7}{2} \rho^{\prime}(1,2)$ (такой способ выбран потому, что $\rho(0,1)=$ $\left.\frac{7}{2} \rho(1,2)\right)$.

Рассмотрим ориентированную триангуляцию $L$ двумерной сферы, содержашую вершину $x$, к которой примыкает $p+q+r+3$ треугольника. Выберем среди треугольников, примыкаюших к вершине $x$, три треугольника $\sigma_{0}, \sigma_{1}$ и $\sigma_{2}$ таких, что при обходе вокруг вершины $x$ по часовой стрелке мы пройдем последовательно через треугольник $\sigma_{0}$, через $r$ других треугольников, через треугольник $\sigma_{1}$, через $p$ других треугольников, через треугольник $\sigma_{2}$ и через $q$ оставшихся треугольников. Обозначим $L_{j}$ комплекс, получаемьй из $L$ при бизвездном преобразовании, ассоциированном с треугольником $\sigma_{j}$. Обозначим через $\alpha_{j}$ цикл, полученньй при коммутировании бизвездных преобразований, ассоциированных с треугольниками $\sigma_{j+1}$ и $\sigma_{j+2}$ комплекса $L$; обозначим через $\alpha_{j}^{\prime}$ цикл, полученньй при коммутировании бизвездных преобразований, ассоциированных с треугольниками $\sigma_{j+1}$ и $\sigma_{j+2}$ комплекса $L_{j}$, где суммы индексов берутся по модулю 3 . Легко проверить, что

$$
\alpha_{0}^{\prime}+\alpha_{1}^{\prime}+\alpha_{2}^{\prime}=\alpha_{0}+\alpha_{1}+\alpha_{2} .
$$


Следовательно,

$$
\begin{aligned}
& \rho^{\prime}(p, q+r+2)+\rho^{\prime}(q, r+p+2)+\rho^{\prime}(r, p+q+2) \\
& \quad=\rho^{\prime}(p, q+r+1)+\rho^{\prime}(q, r+p+1)+\rho^{\prime}(r, p+q+1) .
\end{aligned}
$$

Из этого равенства несложно вывести, что существует константа $\lambda \in \mathbb{Q}$ такая, что $\rho^{\prime}(p, q)=\lambda \rho(p, q)$ для любых $p$ и $q$. Следовательно, $c^{\prime}([\alpha])=\lambda c([\alpha])$ для всех циклов $\alpha$, изображенных на рис. 3 . Аналогично доказывается, что это равенство верно для любого $\alpha \in S$.

Из доказанного утверждения непосредственно следует, что $\operatorname{dim} \widetilde{A} \leqslant 1$. Из сушествования эпиморфизма $\sharp: H^{*}\left(\mathscr{T}^{*}(\mathbb{Q})\right) \rightarrow H^{*}(\mathrm{BPL} ; \mathbb{Q})$ следует, что $\operatorname{dim} H^{4}\left(\mathscr{T}^{*}(\mathbb{Q})\right) \geqslant 1$. $\mathrm{C}$ другой стороны, гомоморфизм $d^{-1} \delta: A \rightarrow \mathscr{T}^{4}(\mathbb{Q})$ индуцирует эпиморфизм $j: \widetilde{A} \rightarrow$ $H^{4}\left(\mathscr{T}^{*}(\mathbb{Q})\right)$. Значит, $\operatorname{dim} \widetilde{A}=\operatorname{dim} H^{4}\left(\mathscr{T}^{*}(\mathbb{Q})\right)=1$. Следовательно, класс когомологий $c$ корректно определен и порождает одномерное векторное пространство $\widetilde{A}$.

14. Знаменатели значений локальных формул. Пусть $f: \mathscr{T}_{n} \rightarrow \mathbb{Q}$ - локальная формула. Выясним, как растут знаменатели значений $f(L)$ с ростом числа вершин триангуляции $L$. Обозначим через $\mathscr{T}_{n, l}$ множество всех ориентированных $(n-1)$-мерных PL-сффер, имеюших не более $l$ вершин. Обозначим через $\operatorname{den}_{l}(f)$ наименьшее обшее кратное знаменателей всех значений $f(L)$, где $L \in \mathscr{T}_{n, l}$. Следуюшие две теоремы дают оценки на рост $\operatorname{den}_{l}(f)$ как функции от $l$.

ТеОРема 14.1. Пусть $\psi \in H^{n}\left(\mathscr{T}^{*}(\mathbb{Q})\right)$ - произвольный класс когомологий. Тогда существуют локальная формула $f$, представляющая класс $\psi$, и натуральная константа $b$ такие, что число $\operatorname{den}_{l}(f)$ делит $b(l+1)$ ! для любого $l$.

ТеОрема 14.2. Пусть $f \in \mathscr{T}^{4}(\mathbb{Q})$ - произвольная локальная формула для первого класса Понтрягина. Тогда число $\operatorname{den}_{l}(f)$ делится на наименьшее общее кратное чисел $1, \ldots, l-3$ для любого четного $l \geqslant 10$.

Теорема 14.1 вьводится из результатов [17] и теоремы 8.1. Теорема 14.2 легко доказывается с помощью явного описания всех локальных формул для первого класса Понтрягина, полученного в п. 11. Из теоремы 14.2 легко вьводится следующее утверждение.

СлЕДСТВИЕ 14.1. Имеет место равенство $H^{4}\left(\mathscr{T}^{*}(G)\right)=0$ для любой собственной подгруппьи $G \subset \mathbb{Q}$.

\section{5. Существование алгоритмов для вычисления локальных формул.}

ТеОРема 15.1. Пусть $\psi \in H^{n}\left(\mathscr{T}^{*}(\mathbb{Q})\right)$ - произвольный класс когомологий. Тогда существует локальная формула $f$, представляющая класс когомологий $\psi$, такая, что задача вычисления значения $f(L)$ по заданной триангулячии $L \in \mathscr{T}_{n}$ алгоритмически разрешима.

ЗАмЕчАНИЕ 15.1. С.П. Новиков (см. [38; с. 166-167]) доказал, что проверка того, является ли симплициальный комплекс $L$ триангулящией $(n-1)$-мерной сферы, - алгоритмически неразрешимая задача при $n \geqslant 6$. Точная формулировка теоремы 15.1 вьглядит следующим образом: существует алгоритм, которьй получает на входе ориентированный симплициальньй комплекс $L$, выдает на выходе значение $f(L)$, если $L \in \mathscr{T}_{n}$, и работает бесконечно долго, если $L \notin \mathscr{T}_{n}$. 
ЗАмечАниЕ 15.2 . Очевидно, что при $n \geqslant 4$ существует кограница $f \in \mathscr{T}^{n}(\mathbb{Q})$, которая не является вычислимой. Поэтому не все рациональные локальные формулы являются вычислимыми.

Автор благодарен В. М. Бухштаберу за постановки задач и постоянное внимание к настоящей работе, а также Л. А. Алания, И. А. Дьнникову, М. Э. Казаряну и А. С. Мищенко за полезные обсуждения.

\section{СПИСОК ЛИТЕРАТУРЫ}

[1] В.А. Рохлин, А. С. Шварц. О комбинаторной инвариантности классов Понтрягина // Докл. АН СССР. 1957. Т. 114. №3. C. 490-493.

[2] R. Thom. Les classes charactéristiques de Pontrjagin des variétés triangulées // Symposium Internacional de Topología Algebraica. Mexico City: La Universidad Nacional Autónoma de Mexico y la UNESCO, 1958. P. 54-67.

[3] A. Ranicki, D. Sullivan. A semi-local combinatorial formula for the signature of a 4k-manifold // J. Differential Geom. 1976. V. 11. № 1. P. 23-29.

[4] H. Whitney. On the theory of sphere-bundles // Proc. Natl. Acad. Sci. USA. 1940. V. 26. № 2. P. 148-153.

[5] А. М. Габриэлов, И. М. Гельфанд, М.В.Лосик. Комбинаторное вычисление характеристических классов. I, II // Функц. анализ и его прил. 1975. Т. 9. № 2. С. 12-28; № 3. C. $5-26$.

[6] А. М. Габриэлов, И. М. Гельфанд, М.В.Лосик. Локальная комбинаторная формула для первого класса Понтрягина // Функц. анализ и его прил. 1976. Т. 10. № 1. С. 14-17.

[7] R. MacPherson. The combinatorial formula of Gabrielov, Gel'fand and Losik for the first Pontrjagin class // Lecture Notes in Math. 1978. V. 677. P. 105-124.

[8] А. М. Габриэлов. Комбинаторные формулы для классов Понтрягина и $G L$-инвариантные цепи // Функц. анализ и его прил. 1978. Т. 12. № 2. С. 1-7.

[9] I. M. Gelfand, R. D. MacPherson. A combinatorial formula for the Pontrjagin classes // Bull. Amer. Math. Soc. (N.S.). 1992. V. 26. № 2. P. 304-309.

[10] J. Cheeger. Spectral geometry of singular Riemannian spaces // J. Differential Geom. 1983. V. 18. № 4. P. 575-657.

[11] M. F. Atiyah, V.K. Patodi, I. M. Singer. Spectral asymmetry and Riemannian geometry // Bull. London Math. Soc. 1973. V. 5. № 2. P. 229-234.

[12] А. А. Гайфуллин. Локальные формулы для комбинаторных классов Понтрягина // Изв. РАН. Сер. матем. 2004. Т. 68. № 5. С. 13-66.

[13] Л. С. Понтрягин. Векторные поля на многообразиях // Матем. сб. 1949. Т. 24. № 2. C. $129-162$.

[14] Л.С. Понтрягин. Некоторые топологические инварианты римановых многообразий // Докл. АН СССР. 1944. Т. 43. № 3. С. 95-98.

[15] Л. С. Понтрягин. Некоторые топологические инварианты замкнутых римановых многообразий // Изв. АН СССР. Сер. матем. 1949. V. 13. № 2. Р. 125-162.

[16] S.S. Chern. Characteristic classes of Hermitian manifolds // Ann. of Math. (2). 1946. V. 47. № 1. P. 85-121.

[17] N. Levitt, C. Rourke. The existence of combinatorial formulae for characteristic classes // Trans. Amer. Math. Soc. 1978. V. 239. P. 391-397.

[18] E. Stiefel. Richtungsfelder und Fernparallelismus in $n$-dimensionalen Mannigfaltigkeiten // Comment. Math. Helv. 1936. V. 8. P. 305-353.

[19] S. Halperin, D. Toledo. Stiefel-Whitney homology classes // Ann. of Math. (2). 1972. V. 96. №3. P. 511-525.

[20] J. Cheeger. A combinatorial formula for Stiefel-Whitney classes // Topology of Manifolds (Proc. Univ. of Georgia, Athens, Ga., 1969). Chicago: Markham, 1970. P. 470-471. 
[21] S.S. Cairns. Triangulated manifolds which are not Brouwer manifolds // Ann. of Math. (2). 1940. V. 41. № 4. P. 792-795.

[22] J. H. C. Whitehead. Note on manifolds // Quart. J. Math. Oxford Ser. (2). 1941. V. 12. № 45. P. 26-29.

[23] S.S. Cairns. Isotropic deformations of geodesic complexes on the 2-sphere and on the plane // Ann. of Math. (2). 1944. V. 45. № 2. P. 207-217.

[24] C.-W. Ho. On certain homotopy properties of some spaces of linear and piecewise linear homeomorphisms. I, II // Trans. Amer. Math. Soc. 1973. V. 181. P. 213-233; 235-243.

[25] В.А.Рохлин. Внутреннее определение характеристических циклов Понтрягина // Докл. AH CCCP. 1952. T. 84. № 3. C. 449-452.

[26] В. М. Бухштабер. Топологические приложения теории двузначных формальных групп // Изв. АН СССР. Сер. матем. 1978. Т. 42. № 1. С. 130-184.

[27] В. М. Бухштабер. Характеристические классы в кобордизмах и топологические приложения теорий однозначных и двузначных формальных групп // Итоги науки и техники. Современные проблемы математики. 1978. Т. 10. С. 5-178.

[28] A. Björner, M. Las Vergnas, B. Sturmfels, N. White, G. Ziegler. Oriented Matroids. Cambridge: Cambridge Univ. Press, 1993. (Encyclopedia Math. Appl. V. 46.)

[29] G. Ziegler. Lectures on Polytopes. New York: Springer-Verlag, 1995. (Grad. Texts in Math. V. 152.)

[30] Р. Том. Некоторые свойства "в целом" дифференцируемых многообразий // Расслоенные пространства. М.: ИЛ, 1958. С. 291-348.

[31] P. L. King. On local combinatorial Pontrjagin numbers. I // Topology. 1977. V. 16. № 1. P. 99-105.

[32] U. Pachner. P.L. homeomorphic manifolds are equivalent by elementary shellings // European J. Combin. 1991. V. 12. № 2. P. 129-145.

[33] В. М. Бухштабер, Т. Е. Панов. Торические действия в топологии и комбинаторике. М.: МЦНМО, 2004.

[34] E. Steinitz, H. Rademacher. Vorlesungen über die Theorie der Polyeder unter Einschluss der Elemente der Topologie. Berlin: Springer-Verlag, 1934. Reprint: Springer-Verlag, 1976.

[35] M. È. Kazarian. The Chern-Euler number of circle bundle via singularity theory // Math. Scand. 1998. V. 82. № 2. P. 207-236.

[36] W. Kühnel, T. F. Banchoff. The 9-vertex complex projective plane // Math. Intelligencer. 1983. V. 5. №3. P. 11-22.

[37] W. Kühnel, G. Lassmann. The unique 3-neighborly 4-manifold with few vertices // J. Combin. Theory Ser. A. 1983. V. 35. № 2. P. 173-184.

[38] И. А. Володин, В. Е. Кузнецов, А. Т. Фоменко. О проблеме алгоритмического распознавания стандартной трехмерной сферы // УМН. 1974. Т. 29. № 5. С. 71-168. 